\title{
Economic and environmental concerns in planning recyclable waste collection systems
}

\author{
Tânia Rodrigues Pereira Ramos ${ }^{\mathrm{a}, *}$, Maria Isabel Gomes ${ }^{\mathrm{b}}$, Ana Paula Barbosa-Póvoa ${ }^{\mathrm{c}}$ \\ a Instituto Universitário de Lisboa (ISCTE-IUL), Business Research Unit (BRU), Av. das Forças Armadas, 1649-026 Lisboa, Portugal \\ ${ }^{\mathrm{b}}$ CMA, Universidade Nova de Lisboa, Campus da Caparica, 2829-516 Caparica, Portugal \\ ' CEG-IST, Instituto Superior Técnico, Universidade de Lisboa, Av. Rovisco Pais, 1049-001 Lisboa, Portugal
}

\section{A R T I C L E I N F O}

\section{Article history:}

Received 18 January 2012

Received in revised form 8 February 2013

Accepted 10 September 2013

\section{Keywords:}

Greenhouse gas emissions

Service areas

Vehicle routing problem

Multiple depots

Multiple products

Recyclable waste collection

\begin{abstract}
A B S T R A C T
This paper addresses the planning of recyclable waste collection systems while accounting for economic and environmental concerns. Service areas and vehicle routes are defined for logistics networks with multiple depots where different products are collected. The problem is modeled as a multi-product, multi-depot vehicle routing problem with two objective functions: distance and $\mathrm{CO}_{2}$ emissions minimization. A decomposition solution method is developed and applied to a real case study. Six scenarios regarding different service areas and objective functions are studied. Savings of up to $22 \%$ in distance and $27 \%$ in $\mathrm{CO}_{2}$ emissions are achieved, exceeding economic and environmental goals.
\end{abstract}

(c) 2013 Elsevier Ltd. All rights reserved.

\section{Introduction}

Recycling involves the processing of used materials into new products, assuming that the product life cycle does not terminate upon product use or consumption. A new cycle then begins in which the primary objective is to recover the remaining value of these products by reinserting them into a forward supply chain (Rubio et al., 2008).

To achieve recycling targets, different collection systems must be built to accommodate the products involved. In Europe, the recycling targets imposed by the European Union have forced member states to develop new collection systems. The traditional routes defined for municipal generic solid waste did not fit the particularities of recyclable materials, for which different vehicles, collection rates and bin locations are required. This context frames the creation of two different waste collection systems-selective and undifferentiated-for recyclable and non-recyclable products. Manufacturers of recyclable products are responsible for providing an adequate destination for their products when they outlast their usefulness. However, this responsibility is often transferred to waste management companies that need to create new collection systems to comply with this responsibility.

The most common recyclable waste collection systems are those related to goods packaging, using materials such as glass, paper, plastic and metal. Different logistics systems can be designed to forward these materials to the recyclers. Variants of recyclable collection systems include systems based on the source separation of each material by the end user or systems based on post-separation at sorting stations. In addition, collection can be based on a single material by noncompartmented vehicles or based on multiple materials using multi-compartmented vehicles. Furthermore, the collection strategies can be based on curbside or drop-off systems, whereas their coverage may be municipal or multi-municipal.

\footnotetext{
* Corresponding author. Tel.: +351 217903412; fax: +351 217903904.

E-mail addresses: tania.ramos@iscte.pt (T.R.P. Ramos), mirg@fct.unl.pt (M.I. Gomes), apovoa@ist.utl.pt (A.P. Barbosa-Póvoa).
} 
Regardless of the variations mentioned above, transportation and sorting always appear as the core activities in managing recyclable waste systems.

Although recycling contributes positively to the environment (see Craighill and Powell (1996) for the environmental benefits of recycling), the activity of collecting recyclable waste is a transportation activity. Therefore, the collection generates greenhouse gas (GHG) emissions (such as $\mathrm{CO}_{2}, \mathrm{CH}_{4}$, $\mathrm{HFCs}$ and $\mathrm{NO}_{x}$ ), resource consumption, land use, acidification, toxic effects on ecosystems and humans, noise and other negative impacts on the environment. Because GHG emissions are quite harmful (particularly $\mathrm{CO}_{2}$ emissions), companies are seeking transportation solutions that minimize $\mathrm{CO}_{2}$ emissions without compromising their economic goals.

The present work addresses the abovementioned concerns and aims to develop the basis for a decision support tool that can aid decision makers in planning recyclable waste collection systems. Specifically, the minimization of variable costs and $\mathrm{CO}_{2}$ emissions are studied. The former is considered as a function of the traveled distance, whereas the latter is assumed as a function of the consumed energy.

This research has been motivated by a real recyclable packaging waste collection system operating in Portugal. The goal is to restructure its current service areas and vehicle routes to decrease the variable costs and $\mathrm{CO}_{2}$ emissions. This case describes the concern of many companies operating in this field and is representative of the decision process in managing recyclable packaging waste collection systems.

Recyclable packaging waste collection systems typically collect three types of packaging materials within a geographic area: paper, glass and plastic/metal (see Fig. 1). Each system provides three types of specific bins scattered over a given area for the consumer to dispose of packaging waste. These materials are then often collected on separate routes because the vehicle fleet is not equipped with compartments. The collection routes are defined for a planning horizon because the recyclable materials have different collection frequencies. Each route is constrained by vehicle capacity and by duration of a working day. Depending on the facilities owned by the system and how the operations are planned, the collected materials can either be consolidated at a transfer station (depot) for later transport or they can be directly unloaded at the sorting station. The transfer stations (depots) act as storage points where the separated packaging materials are stored until there is sufficient quantity to fill a larger truck to be transported to the sorting station. After the sorting operation, the separated materials are baled, compressed and sent to recyclers.

When multiple depots are present, the service areas must be defined to establish the accountability of the different depots at each collection site. As shown in Fig. 1, routes are defined within each service area to collect the three materials. Two types of transportation flows must be considered: the inbound flow from the collection sites to the depots and the outbound flow from the depots to the sorting station. In addition to the distance traveled to collect materials from the collection sites, one must also account for the round-trip distance from the sorting station to the depots.

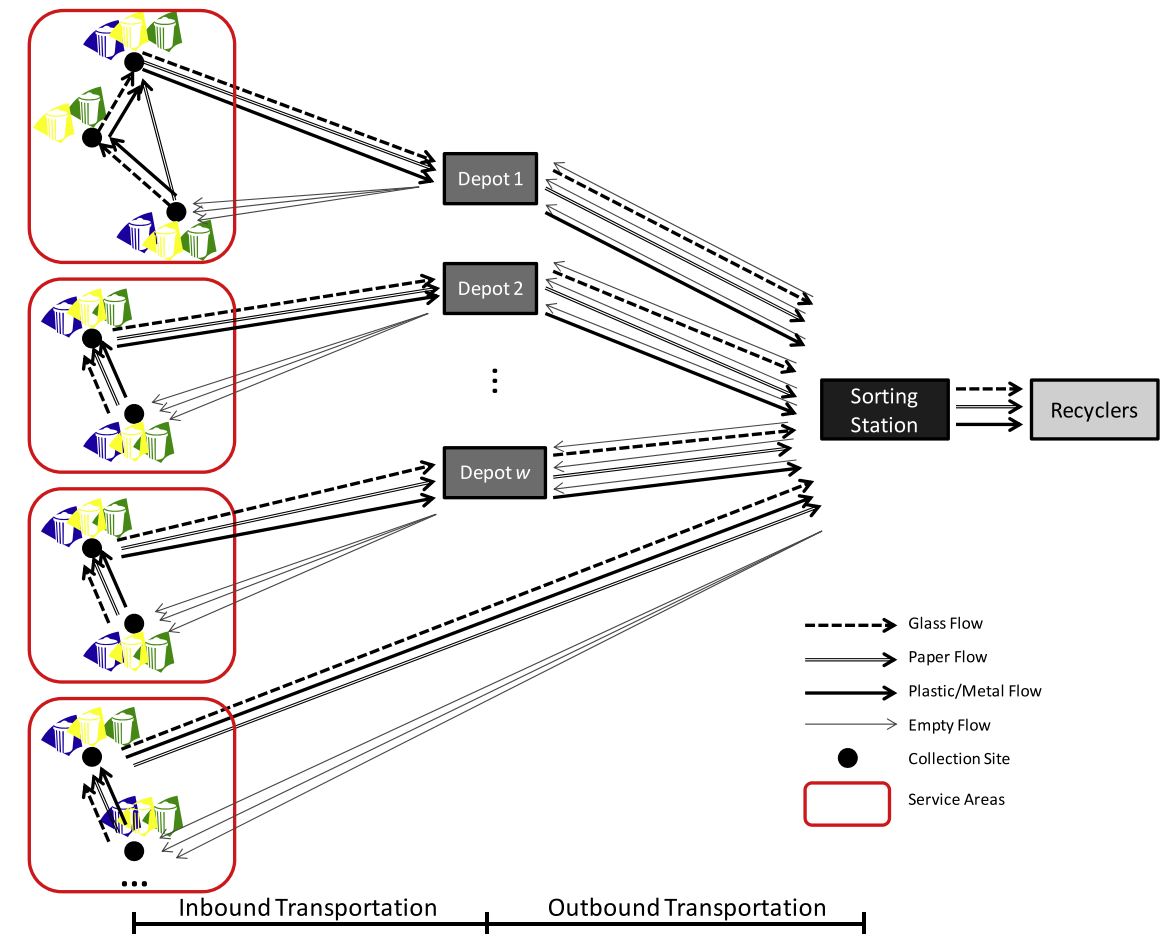

Fig. 1. Schematic representation of a recyclable waste collection network. 
When dealing with a multi-product problem, two alternative solutions can be envisaged regarding the service areas: (a) all products (in this case, recyclable materials) at a collection site are collected by the same depot-each depot has a single service area common to all recyclable materials (Fig. 2(a)); (b) the recyclable materials at each collection site can be collected from different depots-each depot has $|M|$ service areas, where $M$ represents the recyclable materials set (Fig. 2(b)).

Because all materials at each site are collected from the same depot in solution (a) (service areas by depot), this problem is modeled as a multi-product, multi-depot vehicle routing problem (MP-MDVRP). This solution is currently in practice at the company being studied. Because each material can be treated independently in solution (b) (service areas by recyclable material), this problem is modeled as a multi-depot vehicle routing problem (MDVRP) for each material. This approach represents an alternative solution to the current company's practice and is studied in our work.

The MP-MDVRP is modeled as a mixed-integer linear programming (MILP) formulation. This creates two different models to account for the economic and the environmental objectives. A decomposition solution method is proposed to overcome the computational burden observed when addressing large-scale problems.

Tactical decisions such as the delimitation of service areas and the definition of routes in recyclable waste collection systems involving multiple depots, multiple products and multiple transportation flows are studied. The routes definition is considered a tactical decision because the routes are to be maintained for a medium period of time (e.g., 1-6 months) due to the stability of the bin filling rates. Static routes are then studied. The defined routes are to be applied at an operational level and only need to be revised in case of seasonal demands or a significant increase in the disposed quantities by the end user.

Section 2 of this paper presents a literature review on routing problems for multiple depots with environmental concerns and on applications to waste collection systems. Section 3 describes the proposed mathematical formulation for the MPMDVRP. Section 4 discusses the developed decomposition method and tests its effectiveness. The case study application is evaluated in Section 5, in which six scenarios are studied and compared with the company's current solution. In Section 6 , we draw conclusions and discuss ideas for future work.

\section{Literature review}

The multi-depot vehicle routing problem (MDVRP) is a generalization of the vehicle routing problem (VRP) in which, beyond the definition of vehicle routes, it is necessary to determine from which depot customers are to be visited. The MDVRP simultaneously establishes the service areas of each depot and the associated vehicle routes. The vehicle routes are defined such that (1) each route starts and ends at the same depot, (2) each customer is visited exactly once by a vehicle, (3) the total demand of each route does not exceed the vehicle capacity and (4) the total duration of each route (including travel and service time) does not exceed a preset limit. The best solution is typically one that minimizes the total routing cost.

Several models that explore both exact and approximate solutions have been developed for the MDVRP. However, because this is an NP-hard combinatorial problem, the models proposed in the existing literature are predominantly heuristics-based. Few exact algorithms are reported in the literature. Laporte et al. (1984), as well as Laporte et al. (1988), developed exact branch and bound algorithms for solving the symmetric and asymmetric versions, respectively, of the MDVRP. More recently, Baldacci and Mingozzi (2009) developed an exact method for solving the heterogeneous vehicle routing problem (HVRP) that is capable of solving, among other problems, the MDVRP. However, several heuristic algorithms have been proposed to solve MDVRP, including those proposed by Tillman and Cain (1972), Golden et al. (1977), Chao et al. (1993), Renaud et al. (1996), Cordeau et al. (1997), Salhi and Sari (1997), Lim and Wang (2005), Parthanadee and

(a) Service Areas by Depot

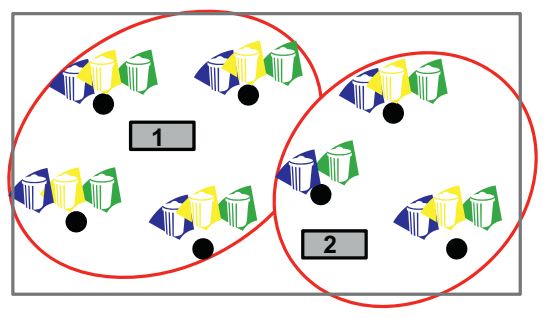

(b) Service Areas by Recyclable Material

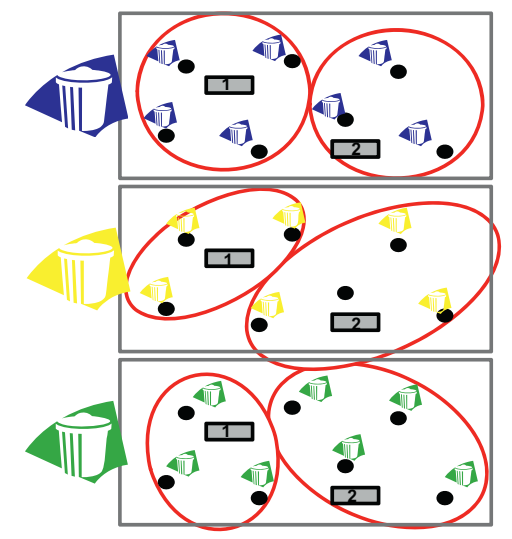

Fig. 2. Illustrative example of (a) service area by depot versus (b) by recyclable material when multiple materials are considered. 
Logendran (2006), Crevier et al. (2007), Pisinger and Ropke (2007), Ho et al. (2008), Zhang et al. (2009) and Dondo and Cerda (2009).

In all of the abovementioned studies, the objective function was defined as either the minimization of the total distance traveled or the minimization of the routing cost. As mentioned in the introduction, companies working in the transportation sector are now concerned with environmental impacts, and this aspect should also be explored within the models. As stated by Sbihi and Eglese (2007), most of the articles published in the VRP field have explored economic objectives. There is scant literature linking VRP models to green logistics issues. Nonetheless, some works exploring these latter aspects have recently been published. Palmer (2007) addressed the role of speed in the reduction of $\mathrm{CO}_{2}$ emissions in a VRP but did not account for the impact of vehicle load. Kara et al. (2007) only considered the weighted load (load multiplied by distance) in the energyminimizing vehicle routing problem. However, the energy consumed by vehicles depends on factors other than the carried load. A comprehensive approach was developed by Bektas and Laporte (2011) in which $\mathrm{CO}_{2}$ emissions are based on a detailed emissions model (see the work of Demir et al. (2011) for a comparative analysis of several vehicle emission models) that accounts for factors such as vehicle load and speed. The authors identified this problem as a pollution-routing problem (PRP). They developed an integer linear programming formulation in which the cost of $\mathrm{CO}_{2}$ emissions and the operational costs of drivers and fuel consumption were minimized. The model was applied to three classes of problems with 10,15 and 20 cities. Ubeda et al. (2011) also examined VRP with respect to environmental concerns and developed a methodology to solve vehicle routing problems with an environmental criterion minimization. This approach was based on the distance traveled and on distance-based emission factors to calculate $\mathrm{CO}_{2}$ emissions. The emission factors depended on an average fuel consumption related to the carried load (empty, low, half, high and full load) multiplied by a conversion factor of $2.61 \mathrm{~kg}$ of $\mathrm{CO}_{2}$ for each liter of diesel consumed. This model was applied to a food distribution case study. More recently, Erdogan and Miller-Hooks (2012) introduced a green vehicle routing problem (G-VRP) that defines routes considering (among its constraints) the fuel level of each vehicle. This work addressed the routing problem of companies that operate an alternative fuel vehicle fleet in which greener fuels such as biodiesel, electricity, ethanol and others could be consumed. The extra challenge of limited refueling infrastructure was considered. Each tour can include stops at one or more alternative fueling stations to allow for vehicles to refuel en route. The G-VRP was formulated as a mixed integer linear program and solved by two constructive heuristics and a customized improvement technique.

The application of routing problems to waste collection systems introduces new characteristics that can lead to different routing problems. Beltrami and Bodin (1974), Tung and Pinnoi (2000), Angelelli and Speranza (2002) and Teixeira et al. (2004) studied a waste collection problem in which the collection sites had different collection frequencies-the periodic vehicle routing problem. In this problem, the collection routes and the day when each one occurs must be defined. Heuristics and meta-heuristics algorithms were developed and applied to undifferentiated and differentiated waste collection systems. When dealing with household waste, the problem is frequently modeled as a capacitated arc routing problem (CARP) in which all arcs in a graph must be visited to allow for waste collection on every street of a city (see Amponsah and Salhi (2004) and Bautista et al. (2008)). For commercial waste collection, the problem is frequently modeled as a VRP with time windows because commercial customers often impose time windows to be respected (see the works of Kim et al. (2006) and Benjamin and Beasley (2010)). McLeod et al. (2011) investigated the benefits of collecting household and commercial waste together rather than in separate collections. It is worth noting that strong efforts have been focused on the design of recycling networks (see, for example, the works of de Figueiredo and Mayerle (2008), Lee and Dong (2008), Paksoy et al. (2011), Schweiger and Sahamie (2013)) rather than on collection routing (Angelelli and Speranza (2002), Teixeira et al. (2004)).

To the best of our knowledge, the multiplicity of depots and, consequently, the definition of service areas, the existence of a transportation flow from depots to the sorting station and the multiplicity of products to be collected have never been addressed. Furthermore, environmental concerns have not yet been considered explicitly in the definition of service areas. Therefore, further work is required to extend these existing works to real situations. In this context, the present paper explores this research opportunity and addresses the multi-product, multi-depot vehicle routing problem with economic and environmental objectives.

\section{Mathematical formulation for the multi-product, multi-depot vehicle routing problem}

A new mathematical formulation for the MP-MDVRP is developed. The formulation is based on the two-commodity flow formulation for the capacitated VRP (CVRP) proposed by Baldacci et al. (2004), in which routes start and end at a single depot. In the present work, the Baldacci et al. (2004) formulation is generalized to model multiple products, multiple depots and inbound and outbound transportation flows. Time duration constraints and different visit frequencies by product are also considered.

The MP-MDVRP is defined through a graph $G=(V, A)$, where $V=\{1, \ldots, n+2 w+s\}$ is the vertex set and $A=\{(i, j): i, j \in V, i \neq j\}$ is the edge set. The vertex set $V$ is partitioned into four subsets $V_{c}=\{1, \ldots, n\}, V_{d}=\{n+1, \ldots, n+w\}$, $V_{f}=\{n+w+1, \ldots, n+2 w\}$ and $V_{s}=\{n+2 w+1, \ldots, n+2 w+s\}$ representing, respectively, the sets of $n$ collection sites, $w$ real depots, $w$ copy depots and $s$ sorting stations. It should be noted that the copy depots set is a replica of the real depots set. This replication is required by the two-commodity flow formulation (see Baldacci et al., 2004) because routes are defined by two paths, one from the real depot to the copy depot and the other from the copy depot to the real one, with 
different starting and ending locations. Each vertex $i$ of $V_{c}$ has a non-negative disposed amount of material $m$ to be collected $\left(p_{i m}\right)$ and a non-negative service duration $t_{i m}$. A distance matrix $D=\left(d_{i j}\right)$ is associated with set $A$. Routes are denoted by set $K$, where $|K|$ is large enough to accommodate the maximal number of routes that the fleet can possibly perform in the planning horizon. Each route $k$ cannot exceed the vehicle capacity $Q_{m}$ or the maximum time allowed for a working day $T$.

The MP-MDVRP that considers two different objective functions (a distance-minimization objective and an energy-minimization objective) leads to two models, the latter being an extension of the former. These models are described below.

\subsection{Distance-minimization MP-MDVRP model}

To formulate the MP-MDVRP with a distance-minimization objective, it is necessary to generalize the flow and binary variables ( $y_{i j}$ and $x_{i j}$, respectively) proposed by Baldacci et al. (2004). Two new indices representing the recyclable materials (index $m$ ) and the routes (index $k$ ) are added. A new variable $\left(z_{i m k}\right)$ is also defined. The model decision variables are thus as follows:

- $x_{i j m k}$, a binary variable that represents the routing solution: $=1$ if site $j$ is visited immediately after site $i$ to collect material $m$ by route $k$; 0 otherwise;

- $y_{i j m k}$, a flow variable that represents the load of material $m$ in route $k$ when traveling from site $i$ to site $j$. The flow $y_{j i m k}$ represents the empty space on the vehicle on route $k$; therefore, $y_{i j m k}+y_{j i m k}=Q_{m}$, where $Q_{m}$ is the vehicle capacity to transport material $m$;

- $z_{i m k}$, a binary variable that assigns collection sites to routes: $=1$ if site $i$, with material $m$, is visited by route $k$; 0 otherwise.

All routes begin at one of the real depots (set $V_{d}$ ) and end at the corresponding copy depot (set $V_{f}$ ). If route $k$ begins at depot $i \in V_{d}$, it must end at the corresponding copy depot of $i$, i.e., depot $i+w \in V_{f}$. Each route is defined by two flow paths: (1) a path defined through variables $y_{i j m k}$, representing the vehicle load on route $k$ that increases along the route as a collecting process is followed; (2) a reverse path defined by variables $y_{j i m k}$, representing the empty space on the vehicle, which decreases along the route (see Fig. 3).

The indices, sets and parameters of this formulation are as follows:

\begin{tabular}{|c|c|}
\hline \multicolumn{2}{|c|}{ Indices } \\
\hline$i, j$ & Node indices \\
\hline$k$ & Route indices \\
\hline$m$ & Recyclable material indices \\
\hline \multicolumn{2}{|l|}{ Sets } \\
\hline$V$ & Node set $V=\{1, \ldots, n+2 w+s\}, V=V_{c} \cup V_{d} \cup V_{f} \cup V_{s}$ \\
\hline$V_{c}$ & Collection site subset $V_{c}=\{1, \ldots, n\}$ \\
\hline$V_{d}$ & Real depot subset $V_{d}=\{n+1, \ldots, n+w\}$ \\
\hline$V_{f}$ & Copy depot subset $V_{f}=\{n+w+1, \ldots, n+2 w\}$ \\
\hline$V_{s}$ & Sorting station subset $V_{s}=\{n+2 w+1, \ldots, n+2 w+s\}$ \\
\hline$K$ & Routes set $K=\{1, \ldots, r\}, K=K_{1} \cup \ldots \cup K_{i}$ \\
\hline$K_{i}$ & Routes belonging to depot i \\
\hline$M$ & Recyclable material set $M=\{1, \ldots, h\}$ \\
\hline \multicolumn{2}{|c|}{ Parameters } \\
\hline$d_{i j}$ & Distance between collection sites $i$ and $j$ (in meters) \\
\hline$p_{i m}$ & Amount of material $\mathrm{m}$ to be collected at collection site $i$ (in $\mathrm{kg}$ ) \\
\hline$c_{i m}$ & Number of bins of material $m$ at collection site $i$ \\
\hline$f r_{m}$ & Collection frequency of material $m$ within the timeframe \\
\hline$Q_{m}$ & Vehicle capacity to transport material $m$ in inbound transportation (in $\mathrm{kg}$ ) \\
\hline$Q T_{m}$ & Vehicle capacity to transport material $m$ in outbound transportation (in $\mathrm{kg}$ ) \\
\hline$v b$ & Speed between collection sites and between collection sites and the depots (in meters per minute) \\
\hline$v w$ & Speed within collection sites (in meters per minute) \\
\hline$U$ & Amount of time to collect a bin (in minutes) \\
\hline$S$ & Average distance between bins within a collection site (in meters) \\
\hline$L$ & Time to unload the vehicle in the end of a route at the depot (in minutes) \\
\hline$t_{i m}$ & Time required to collect site $i$ with material $m$ (in minutes) $t_{i m}=c_{i m}\left(U+\frac{s}{v w}\right)$ \\
\hline$b_{i j}$ & Time to travel from site $\mathrm{i}$ to site $\mathrm{j}$ (in minutes) $b_{i j}=\frac{d_{i j}}{v b}$ \\
\hline$T$ & Maximum time allowed for a working day (in minutes) \\
\hline
\end{tabular}




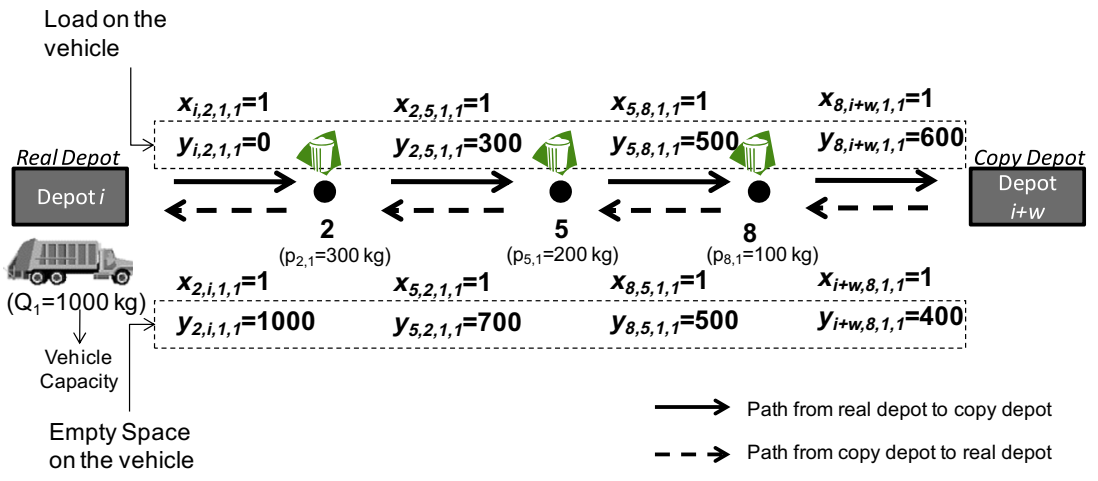

Fig. 3. Illustration of the two paths defining a route by the two-commodity flow formulation.

Model

$$
\begin{aligned}
& \operatorname{Min} D=\frac{1}{2} \sum_{i \in V} \sum_{j \in V} \sum_{m \in M} \sum_{k \in K} x_{i j m k} d_{i j} f r_{m}+\sum_{m \in M} \sum_{i \in V_{c}} c_{i m} S f r_{m}+2 \sum_{i \in V_{c}} \sum_{j \in V_{f}} \sum_{m \in M} \sum_{k \in K_{j}} \sum_{h \in V_{s}} \frac{y_{i j m k} f r_{m}}{Q T_{m}} d_{h j} \\
& \text { subject to } \sum_{j \in V}\left(y_{i j m k}-y_{j i m k}\right)=2 p_{i m} z_{i m k} \quad \forall i \in V_{c}, \forall k, \forall m \\
& \sum_{i \in V_{c}} \sum_{j \in V_{f}} \sum_{k \in K} y_{i j m k}=\sum_{i \in V_{c}} p_{i m} \quad \forall m \\
& \sum_{i \in V_{c}} \sum_{j \in V_{f}} \sum_{k \in K} y_{j i m k} \leqslant|K| Q_{m}-\sum_{i \in V_{c}} p_{i m} \quad \forall m \\
& \sum_{j \in V_{c}} y_{j i m k} \leqslant Q_{m} \quad \forall i \in V_{d}, \forall k \in K_{i}, \forall m \\
& \sum_{i \in V_{c}} y_{j i m k}=0 \quad \forall j \in V_{d}, \forall k, \forall m \\
& \sum_{i \in V} X_{i j m k}=2 z_{j m k} \quad \forall j \in V_{c}, \forall k, \forall m \\
& y_{i j m k}+y_{j i m k}=Q_{m} x_{i j m k} \quad \forall i \in V, \forall j \in V, \forall k, \forall m \\
& \sum_{k \in K} z_{i m k}=1 \quad \forall i \in V_{c}, \forall m \\
& y_{i j m k} \leqslant B i g M z_{i m k} \quad \forall i \in V_{c}, \forall j \in V, \forall k, \forall m \\
& z_{\text {imk }}=z_{(i+w) m k} \quad \forall i \in V_{d}, \forall k \in K_{i}, \forall m \\
& \sum_{i \in V} \sum_{j \in V} t_{i m} x_{i j m k}+\sum_{i \in V} \sum_{j \in V} b_{i j} x_{i j m k}+L \leqslant 2 T \quad \forall k, \forall m \\
& \sum_{j \in V_{c}} x_{i j m k} \leqslant 1 \quad \forall i \in V_{d}, \forall k \in K_{i}, \forall m \\
& \sum_{i \in V_{c}} x_{i j m k}=0 \quad \forall j \in V_{f}, \forall k \notin K_{j}, \forall m \\
& \sum_{j \in V_{c}} x_{i j m k}=0 \quad \forall i \in V_{d}, \forall k \notin K_{i}, \forall m \\
& \sum_{j \in V} \sum_{k \in K_{h}} x_{i j m k}-\sum_{j \in V} \sum_{k \in K_{h}} x_{i j(m+1) k}=0 \quad \forall i \in V_{c}, \forall h \in V_{d}, \forall m \\
& y_{i j m k} \geqslant 0 \quad \forall i, j \in V, k \in K, m \in M \\
& x_{i j m k} \in\{0,1\} \quad \forall i, j \in V, k \in K, m \in M \\
& z_{\text {imk }} \in\{0,1\} \quad \forall i \in V_{c}, k \in K, m \in M
\end{aligned}
$$

The objective function, Eq. (1), models the minimization of the total distance traveled, which includes the inbound transportation (first and second terms) and the outbound transportation (third term). The inbound transportation considers the distance from the collection sites to the depots where the collection routes are defined by two paths. Thus, each solution edge is counted twice, doubling the distance traveled. To identify the actual value, the distance must be divided by two to eliminate the value of the second path. The second term is related to the distance traveled within the collection sites. Because the collection sites correspond to localities that may have scattered bins, this term is required to account for the traveled distance within the localities. The third term models the round-trip distance between the sorting station and the depots. 
Constraint (2) states that the outflow minus the inflow at each collection site is equal to twice the amount to be collected. Constraints (3) to (6) model the inflows and outflows of the real and copy depots. For the real depots, the total outflow will be zero because all vehicles are empty when leaving the real depots (Constraint 6) and the total inflow of each real depot corresponds to the capacity of the used routes based on that depot (Constraint 5). For the copy depots, the total inflow is equal to the total amount to be collected (Constraint 3 ) and the outflow corresponds to the residual capacity of the routes used (Constraint 4). Constraint (7) guarantees that any feasible solution contains two edges incident to each collection site due to the two paths that characterize each route. The vehicle capacity and duration constraints are modeled by Eqs. (8) and (12), respectively. Because each collection site with material $m$ must be visited by only one route, Constraint (9) guarantees this condition. Constraint (10) ensures that if collection site $i$ is not visited by route $k$, then the corresponding flow variable $y_{i j m k}$ is set to zero. Constraint (11) matches the real depots with the copy depots, ensuring that when a route begins at a real depot it will end at the corresponding copy depot. Constraint (13) ensures that each route will leave its home depot at most once. Constraints (14) and (15) jointly ensure that a route cannot leave and return to a depot other than its home depot (real and copy depot). Constraint (16) defines service areas by depot, i.e., guarantees that all recyclable materials at each collection site are collected from the same depot. Finally, the domains of all variables are given by Constraints (17) to (19).

The MP-MDVRP is a generalization of the MDVRP. To model the MDVRP, Constraint (16) is removed, allowing for the routes of each recyclable material to be determined independently.

\subsection{Energy-minimization MP-MDVRP model}

To incorporate environmental concerns into the MP-MDVRP model, an energy minimization objective is chosen. When a vehicle travels over an arc $(i, j)$, it emits a certain amount of GHG. This depends on the fuel consumption, which in turn, is directly connected with the energy requirements. If the required energy is minimized, the GHG amount will consequently be minimized.

The energy requirements are established through fuel consumption that is a function of several aspects such as vehicle load (curb weight plus load), speed, road slope, engine features, vehicle frontal surface area, coefficients of rolling resistance and drag, and air density. These aspects are then considered in the calculation of the energy required (in kilojoules) to travel over an arc $(i, j)$ as expressed in Eq. (20) (originally proposed by Barth et al. (2004) and later adapted by Bektas and Laporte (2011)):

$$
E_{i j}=R N B\left(d_{i j} / v_{i j}\right)+\left(P_{i j} / \varepsilon+P_{a}\right) / \eta / 1000
$$

where $R$ is the engine friction factor (in $\mathrm{kJ} / \mathrm{rev} /$ liter, representing the fuel energy used at zero power output to overcome engine friction per engine revolution and unit of engine displacement), $N$ is the engine speed in revolutions per second (rev/s), $B$ is the engine displacement in liters, $d_{i j}$ is the distance between site $i$ and $j$ (in meters), $v_{i j}$ is the speed on arc ( $i, j$ ) in meters per second, $P_{i j}$ is the total tractive power demand at the wheels to travel over arc $(i, j)$ (in joules), $\varepsilon$ is vehicle drivetrain efficiency, $P_{a}$ is the engine power requirement for accessories such as air conditioning (which from now on will be considered null, i.e., $\left.P_{a}=0\right)$ and $\eta$ is engine efficiency.

Following the approach of Bektas and Laporte (2011), $P_{i j}$ is given by Eq. (21), where $\alpha$ and $\beta$ are the arc and vehicle specific constants, respectively:

$$
P_{i j}=\alpha\left(c w+\mu_{i j}\right) d_{i j}+\beta v_{i j}^{2} d_{i j}
$$

The first term of $P_{i j}$ is referred to as the load-induced energy requirements: $\alpha=u+g \sin \theta+g C_{r} \cos \theta$, where $u$ is the acceleration (in $\left.\mathrm{m} / \mathrm{s}^{2}\right), g$ is the gravitational constant $\left(9.81 \mathrm{~m} / \mathrm{s}^{2}\right), \theta$ is the road slope in degrees (we are assuming in Eq. (21) that all arcs have the same road slope), $C_{r}$ is the coefficient of rolling resistance, $c w$ is the curb weight and $\mu_{i j}$ is the load carried by the vehicle on $\operatorname{arc}(i, j)$. The second term of Eq. (21) is called the speed-induced energy requirements: $\beta=0.5 C_{d} O \rho$, where $C_{d}$ is the drag coefficient, $O$ is the frontal surface area of the vehicle (in $\mathrm{m}^{2}$ ) and $\rho$ is the air density (in $\mathrm{kg} / \mathrm{m}^{3}$ ). Because we considered two types of transportation flows (inbound and outbound) operated by different vehicles, we will have different values for the vehicle specific constant $\beta$. Assume $\beta_{\text {inb }}$ for the vehicles used in the inbound transportation and $\beta_{\text {outb }}$ for the vehicles used in the outbound transportation. The same distinction applies to parameters $c w\left(c w_{\text {inb }}\right.$ and $\left.c w_{\text {outb }}\right)$ and $B\left(B_{\text {inb }}\right.$ and $B_{\text {outb }}$ ).

Considering the parameters associated with each type of vehicle, Expressions (22) and (23) compute the aggregate parameters $\xi_{\text {inb }}$ and $\xi_{\text {outb }}$, representing the energy required to travel a meter (in kilojoules per meter) for the inbound and outbound vehicles, respectively. We assume speed $\left(v_{i j}\right)$ as a parameter and not as a decision variable because average speeds within collection sites $(v w)$ and between collection sites $(v b)$ are considered representative of the truck speed.

$$
\begin{aligned}
& \xi_{\text {inb }}=\frac{R N B_{\text {inb }}}{v b / 60}+\frac{\alpha c w_{\text {inb }}+\beta_{\text {inb }}(v b / 60)^{2}}{\eta \varepsilon 1000} \\
& \xi_{\text {outb }}=\frac{R N B_{\text {outb }}}{v b / 60}+\frac{\alpha c w_{\text {outb }}+\beta_{\text {outb }}(v b / 60)^{2}}{\eta \varepsilon 1000}
\end{aligned}
$$

Eq. (24) translates the energy requirements $E_{i j}$ into fuel requirements $F_{i j}$ (in liters), given $\phi$ as the fuel-to-air equivalence ratio, $f d$ as the fuel density (g/l) and $43.2 \mathrm{~kJ} / \mathrm{g}$ as the lower heating value of typical diesel fuel (Barth et al., 2004), 


$$
F_{i j}=\phi \cdot E_{i j} /(43.2 \cdot f d)
$$

To convert the fuel requirements into $\mathrm{CO}_{2}$ emissions, we assume a conversion factor of one liter of diesel fuel containing $2.672 \mathrm{~kg}$ of $\mathrm{CO}_{2}$ (Carbon Trust, 2010).

Based on the abovementioned considerations, and to formulate the MP-MDVRP with an energy-minimization objective, we must add two auxiliary variables $\left(\delta_{i j}\right.$ and $\left.\mu_{i j}\right)$ to track the path between the real and copy depots. In the proposed formulation the route solution is given by two flow paths. Thus, the auxiliary variables will isolate the load path such that it will be correctly accounted in the objective function. Note that variable $y_{i j m k}$ provides the carried load and the empty space for each $\operatorname{arc}(i, j)$.

The MP-MDVRP with environmental concerns is thus the minimization of the total energy required for inbound and outbound transportation, derived from Eq. (20). This objective function contains three components: the inbound transportation between collection sites and depots (25a), the inbound transportation within collection sites (25b) and the outbound transportation (25c).

$$
\begin{aligned}
\text { Min } E= & \xi_{\text {inb }} \\
2 & \sum_{i \in V} \sum_{j \in V} \sum_{m \in M} \sum_{k \in K} x_{i j m k} d_{i j} f r_{m}+\sum_{i \in V} \sum_{j \in V} \sum_{m \in M} \sum_{k \in K} \frac{\alpha \mu_{i j m k} d_{i j} f r_{m}}{\eta \varepsilon 1000} \\
& +\sum_{i \in V} \sum_{j \in V_{c}} \sum_{m \in M} \sum_{k \in K} \frac{\alpha \mu_{i j m k} c_{j m} S f r_{m}}{\eta \varepsilon 1000}+\sum_{i \in V_{c}} \sum_{m \in M} \pi_{i m} \\
& +\sum_{i \in V_{c} j \in V_{f}} \sum_{m \in M} \sum_{k \in K_{j}} \sum_{h \in V_{s}} \frac{y_{i j m k} f r_{m}}{Q T_{m}} d_{h j}\left(\frac{\alpha Q T_{m}}{\eta \varepsilon 1000}+2 \xi_{\text {outb }}\right)
\end{aligned}
$$

The energy required to travel within each collection site considers the vehicle load when entering a site (first term of (25b)) and the distance to travel within a collection site to collect all containers (parameter $\pi_{i m}$, see Expression (26)). It should be noted that, in the latter, the load picked up in each container is taken into account.

$$
\pi_{i m}=c_{i m} S f r_{m}\left(\frac{R N B_{i n b}}{\nu w / 60}+\frac{\alpha c w_{i n b}+\alpha \frac{p_{i m}}{c_{i m}}\left(\frac{c_{i m}+1}{2}\right)+\beta_{i n b}(v w / 60)^{2}}{\eta \varepsilon 1000}\right)
$$

In addition to Constraints (2) through (19) defined in the previous model, the current model requires eight more constraints to describe the load carried by the routes in inbound transportation:

$$
\begin{aligned}
& \sum_{j \in V} \mu_{i j m k}-\sum_{j \in V} \mu_{j i m k}=p_{i m} z_{i m k} \quad \forall i \in V_{c}, \forall m, \forall k \\
& p_{i m} \delta_{i j m k} \leqslant \mu_{i j m k} \leqslant Q_{m} \delta_{i j m k} \quad \forall i, j, m, k \\
& \sum_{i \in V_{d} j \in V_{c}} \sum_{m \in M} \sum_{k \in K} \delta_{i j m k} \leqslant|K| \\
& \sum_{i \in V_{f}} \sum_{j \in V_{c}} \sum_{m \in M} \sum_{k \in K} \delta_{j i m k} \leqslant|K| \\
& \delta_{i j m k} \leqslant x_{i j m k} \quad \forall i, j, m, k \\
& \mu_{i j m k} \leqslant y_{i j m k} \quad \forall i, j, m, k \\
& \delta_{i j m k}, \mu_{i j m k} \geqslant 0 \quad \forall i, j \in V, k \in K, m \in M
\end{aligned}
$$

Constraint (27) represents the flow balance, where the outflow minus the inflow at each collection site is equal to the amount to be collected. The load carried in the route is bounded by Constraint (28). Constraints (29) and (30) state that a maximum of $|K|$ routes may depart from real depots and arrive at copy depots. The auxiliary variables are linked to the main variables through Constraints (31) and (32). The auxiliary variables domain is given in Constraint (33).

\section{Decomposition solution method to solve the multi-product, multi-depot vehicle routing problem}

\subsection{Description}

When solving large instances in preliminary tests through the proposed mathematical models, computational difficulties were faced due to the hard combinatorial problem characteristics. Only instances with up to 12 sites and 2 depots could be solved to optimality. For the real case study, the commercial solver CPLEX was not capable of generating any of the two monolithic models described. To overcome this problem, a decomposition solution method was developed. In the MPMDVRP, two main decisions must be simultaneously made: which depot collects each site (considering concurrently all materials at each site) and the collection sequence. The solution method then decomposes the problem into these two main decisions. The first step defines the service areas for each depot and the second step defines the final collection routes. 
In the first step, service areas are firstly defined for each material by solving a MDVRP with Mixed Closed and Open Routes (MDVRP-MCO) for all collection sites. The MDVRP-MCO is a relaxation of the original problem (the MP-MDVRP), in which just one product has to be collected and the vehicles are not restricted to start and finish at the same depot (closed routes), if the objective function is minimized. As a result, closed and open routes (the starting and ending depot is different) are produced. Given that the original problem only allows closed routes, when open routes are obtained, a MDVRP is solved considering a subset of the collection sites, the ones belonging to open routes. As result, only closed routes are obtained which establishes the service area for each depot. Both MDVRP-MCO and MDVRP are solved as many times as the number of different materials considered in the problem. At this point, we will have $|M|$ different service areas (service areas by recyclable material). Since a single service area by depot is envisaged, a heuristic procedure is applied to allocate each collection site to a single depot. In the second step, a VRP is solved for each depot and material to define the final collection routes.

Each step of the decomposition method is described below in greater detail.

\section{Step 1: Service areas definition}

Step 1 allows for the study of two different service area configurations, service areas by depot and by recyclable material. This step involves two mathematical formulations (from now called formulations 1.1 and 1.2) and one heuristic procedure (called procedure 1.3).

It begins defining the service areas for each material $m \in M$ by solving a relaxation of the original problem that is simpler and easier to solve with commercial solvers: the MDVRP-MCO. The MDVRP-MCO (formulation 1.1) is considered here because its mathematical formulation is capable of solving large instances (see the work of Ramos et al. (2013)). On the other hand, a significant portion of the MDVRP-MCO solution is feasible for the original problem (i.e., all the closed routes). For the open routes defined by the MDVRP-MCO, a second mathematical formulation is solved to close the routes - the MDVRP formulation. Only the sites belonging to each open route are used as input data for the MDVRP (formulation 1.2), meaning that small instances are solved. By solving the MDVRP, these sites are naturally assigned to depots because the MDVRP solution only defines closed routes.

Table 1 shows the two formulations for the MDVRP-MCO: the distance minimization (at left) and the energy minimization (at right). Both are solved independently for each material. Only the node set $V$ is considered (the route set $K$ and the recyclable material set $M$ are not considered). An additional parameter $\lambda$ is included, representing the maximum number of routes allowed. Variable $z$ is unnecessary in these formulations, and two new variables are added, $a_{i j}$ and $e_{i j}$, to cope with the time duration constraints for each route. Some equations are the same as those proposed for the MP-MDVRP, but they were adjusted to cope with a single set (set $V$ ). Three extra equations are added: Constraints (34) and (35) address the number of routes ( $\lambda$ ), and Constraint (36) guarantees the same number of routes beginning and ending at each depot. It should be noted that because open routes can be part of the solution, the number of routes beginning at a given depot may be different from the number of routes that end at that depot.

Table 2 shows the formulations for the MDVRP. The formulations are solved for each open route produced by formulation 1.1. Set $M$ is not considered, and the set of collection sites is reduced to the sites belonging to open routes. Because now route set $K$ is included, the decision variables $a_{i j}$ and $e_{i j}$ are no longer necessary for controlling route duration.

After solving formulations 1.1 and 1.2 for each material $m$, the service areas are defined by material. If service areas by depot are required, heuristic procedure 1.3 is run to ensure that all materials at each collection site are collected from the same depot.

The heuristic procedure starts by identifying from the output of formulations 1.1 and 1.2 , which depot collects each material from each collection site. Then, for each site, it is assessed if a unique depot is responsible for its three materials. If not, that site is classified as unclear. The assignment of the unclear sites to depots is then set according to the material with the highest collection frequency given that its contribution to the objective function is stronger than that of the other materials. However, collection sites may not have all the materials to be collected. If so, the assignment should be made according to the second highest collection frequency material, and so on.

The chosen assignment rule is sustained by a comparative study performed with test instances (described in Section 4.2) in which the service areas proposed by the MDVRP for each material are compared with those proposed by the MP-MDVRP (both monolithic models are solved through the commercial solver CPLEX). In all test instances, the same number of materials and collection frequencies found in the motivation case study are assumed-that is, three materials $\left(m_{1}, m_{2}, m_{3}\right)$ and frequencies $\left(f r_{m 1}=1, f r_{m 2}=4, f r_{m 3}=2\right)$.

The service areas obtained for the MP-MDVRP match, in 12 out of 15 instances, with the service areas provided when solving the MDVRP for the material with the highest collection frequency $\left(f r_{m 2}=4\right)$. This result is explained by the fact that the $m_{2}$ collection contribution to the total traveled distance (or for the total required energy) is four times larger than $m_{1}$ and two times larger than $m_{3}$.

Fig. 4 illustrates these results for one test instance (instance 8). This instance is solved by the MDVRP distance-minimization model for each material and by the MP-MDVRP distance-minimization model. It can be observed that by applying the MDVRP model by material (Fig. 4(b)), different service areas are produced. In comparing the three service areas with the one produced by the MP-MDVRP model (Fig. 4(a)), it is observed that the latter is the same as the $m_{2}$ service area.

In our case study, there is a significant difference between the collection frequencies of each material such that one material stands out from the others. In this case, using the material with the highest collection frequency as the assigning rule 
Table 1

Formulation 1.1 - MDVRP-MCO.

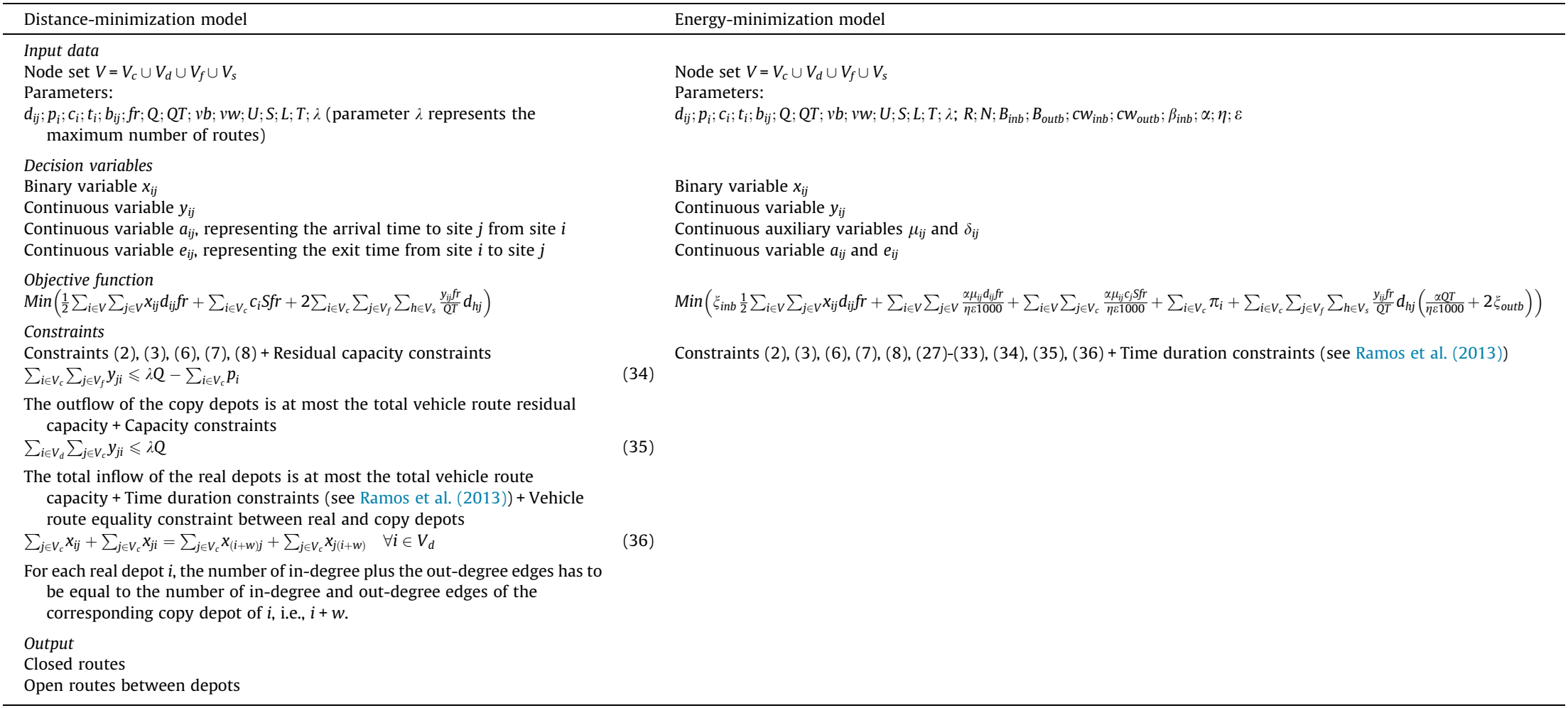


Table 2

Formulation 1.2 - MDVRP.

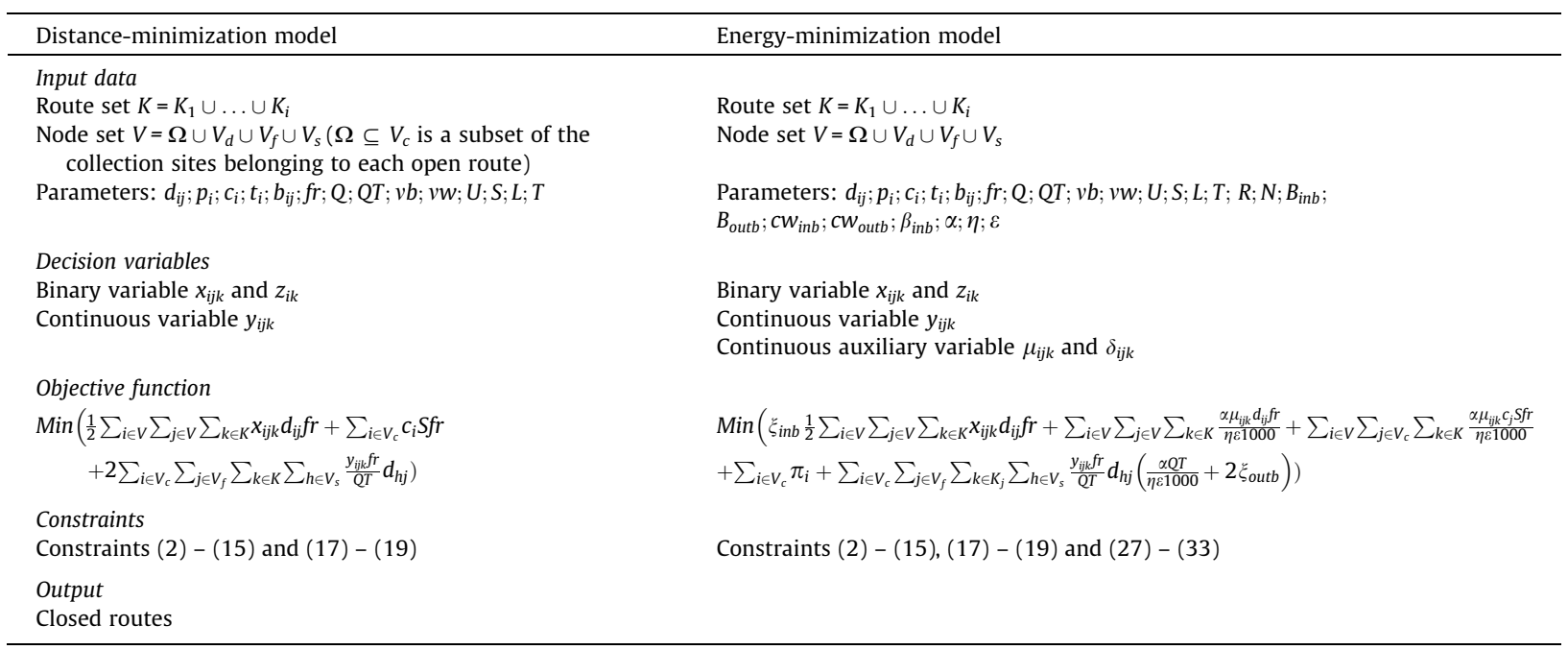

provides good results. In $80 \%$ of the tested instances, it provided the same solution as that obtained by solving a MP-MDVRP. In cases in which no material stands out from the others, different assigning rules must be investigated, such as site-by-site assigning to the nearest service area through an iterative process, or assigning to the nearest depot, or using the MP-MDVRP model only with the unclear sites or any other reasonable rule.

\section{Step 2: Routes definition}

The routes designed at Step 1 can be improved when the final service areas are established (the output of Step 1 ). Step 2 solves a vehicle routing problem for each depot and for each recyclable material so that the final collection routes are defined. The mathematical formulation used to model the VRP in Step 2 is based on the two-commodity flow formulation (Baldacci et al., 2004), in which time duration constraints are added. The formulations applied for the economic (distance minimization) and the environmental (energy minimization) objectives are shown in Table 3.

\section{(a) Solving the MP-MDVRP distance-minimization model}

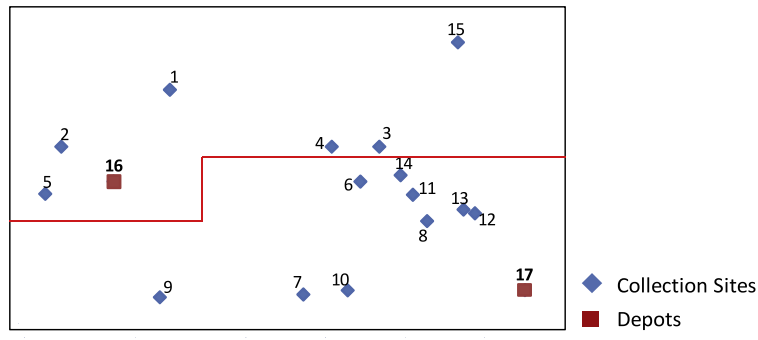

(b) Solving the MDVRP distance-minimization model for each material
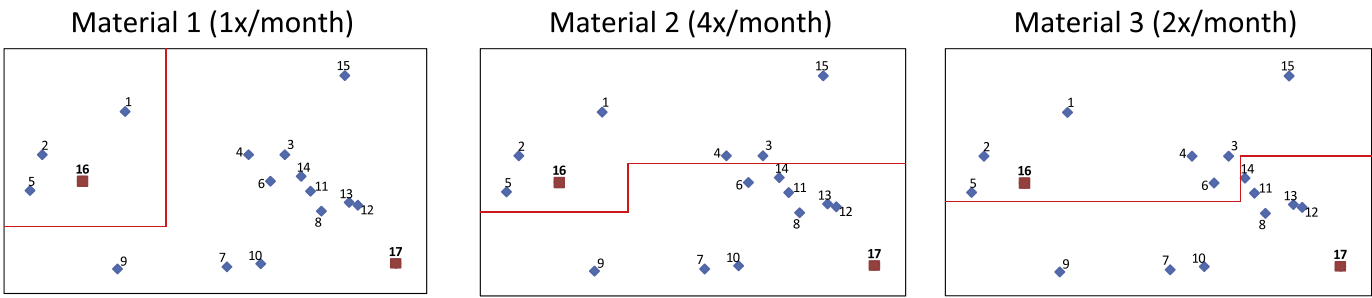

Fig. 4. Service area results for test instance 8 obtained by (a) solving the MD-MDVRP model versus (b) the MDVRP model for each material. 
Table 3

Formulation 2 - VRP.

\begin{tabular}{|c|c|}
\hline Distance-minimization model & Energy-minimization model \\
\hline $\begin{array}{l}\text { Input data } \\
\text { Node set } V=V_{c} \cup V_{d} \cup V_{f} \cup V_{s} \text { where }\left|V_{d}\right|=1 \text { and }\left|V_{f}\right|=1 \\
\text { Parameters: } d_{i j} ; p_{i} ; c_{i} ; t_{i} ; b_{i j} ; f r ; Q ; Q T ; v b ; v w ; U ; S ; L ; T ; \lambda\end{array}$ & $\begin{array}{l}\text { Node set } V=V_{c} \cup V_{d} \cup V_{f} \cup V_{s} \text { where }\left|V_{d}\right|=1 \text { and }\left|V_{f}\right|=1 \\
\text { Parameters: } d_{i j} ; p_{i} ; c_{i} ; t_{i} ; b_{i j} ; f r ; Q ; Q T ; v b ; v w ; U ; S ; L ; T ; \lambda ; R ; N ; B_{\text {inb }} \text {; } \\
B_{\text {out }} ; c w_{\text {inb }} ; c w_{\text {outb }} ; \beta_{\text {inb }} ; \alpha ; \eta ; \varepsilon\end{array}$ \\
\hline $\begin{array}{l}\text { Decision variables } \\
\text { Binary variable } x_{i j} \\
\text { Continuous variable } y_{i j}, a_{i j} \text { and } e_{i j}\end{array}$ & $\begin{array}{l}\text { Binary variable } x_{i j} \\
\text { Continuous variable } y_{i j}, a_{i j} \text { and } e_{i j} \\
\text { Continuous auxiliary variable } \mu_{i j} \text { and } \delta_{i j}\end{array}$ \\
\hline $\begin{array}{l}\text { Objective function } \\
\operatorname{Min}\left(\frac{1}{2} \sum_{i \in V} \sum_{j \in V} x_{i j} d_{i j} f r+\sum_{i \in V_{c}} c_{i} S f r+2 \sum_{i \in V_{c}} \sum_{j \in V_{f}} \sum_{h \in V_{s}} \frac{y_{i j} f r}{Q T} d_{h j}\right)\end{array}$ & $\begin{array}{l}\operatorname{Min}\left(\xi_{\text {inb }} \frac{1}{2} \sum_{i \in V} \sum_{j \in V} x_{i j} d_{i j} f r+\sum_{i \in V} \sum_{j \in V} \frac{\alpha \mu_{i j} d_{i j} f r}{\eta \xi 1000}+\sum_{i \in V} \sum_{j \in V_{c}} \frac{\alpha \mu_{i j} c_{j} S f r}{\eta \varepsilon 1000}+\sum_{i \in V_{c}} \pi_{i}\right. \\
\left.+\sum_{i \in V_{c}} \sum_{j \in V_{f}} \sum_{h \in V_{s}} \frac{y_{i j} f r}{Q T} d_{h j}\left(\frac{\alpha Q T}{\eta \varepsilon 1000}+2 \xi_{\text {outb }}\right)\right)\end{array}$ \\
\hline $\begin{array}{l}\text { Constraints } \\
\text { Constraints (2), (3), (6), (7), (8), (34), (35) + Time duration } \\
\text { constraints }\end{array}$ & $\begin{array}{l}\text { Constraints (2), (3), (6), (7), (8), (27)-(33), (34), (35) + Time duration } \\
\text { constraints }\end{array}$ \\
\hline $\begin{array}{l}\text { Output } \\
\text { Closed routes }\end{array}$ & \\
\hline
\end{tabular}

\subsection{Effectiveness tests}

In this section, we evaluate the effectiveness of the proposed decomposition method by applying it to a set of test instances. The results are then compared with the optimal solutions from the monolithic models presented at Section 3. Due to the complexity of the MP-MDVRP and MDVRP, the instances that can be solved to optimality are typically of small size. Therefore, fifteen small instances (based on the characteristics of the case study) were randomly generated because the existing literature has not identified any instances fitting our problem. These fifteen instances consider three recyclable materials and two depots, with the exception of instances 13 and 15, which have three depots. The number of collection sites $\left(\left|V_{c}\right|\right)$ varies from six to twenty, as shown in the second column of Table 4.

The MILP formulations developed both for the monolithic model and the decomposition method were implemented in GAMS 23.6 and solved using the CPLEX Optimizer 12.1 .0 on an Intel Xeon CPU X5680 @ $3.33 \mathrm{GHz}$. In this testing, the branch-and-bound computation time was limited to $8 \mathrm{CPU}$ hours.

Because the decomposition solution method can define service areas by recyclable material and by depot, both cases are tested. The objective function considered is the distance-minimization function. Therefore, the MP-MDVRP monolithic model proposed in Section 3.1 is used. The results obtained by solving the MDVRP (service areas by recyclable material) and MP-MDVRP (service areas by depot) by the monolithic model and by the decomposition method are presented in Table 4.

A percentage deviation between the solution values and the CPU time determined by the monolithic model and the decomposition method are computed. Regarding the Service Areas by Recyclable Material, we can see from the Deviation column of Table 4 that the decomposition method achieves the optimal solution in 7 out of 15 instances (deviation equal to $0 \%$ ) and that the solution deviation is less than $1 \%$ in 6 instances. The average objective function value (OFV) deviation is $0.3 \%$, and the time required for the decomposition method is approximately $90 \%$ less than that required by the monolithic model. Regarding the Service Areas by Depot, the decomposition method finds the same solution as the monolithic model in 10 out of 15 instances, with an average percentage of OFV deviation of approximately $0.5 \%$. The monolithic model proves the optimality in only 5 instances within the time limit of $8 \mathrm{~h}$. With respect to CPU time, the average deviation of the decomposition method is $99 \%$ less than that of the monolithic model.

The effectiveness tests are also used to evaluate the lower and upper bounds provided by the decomposition method. The MDVRP-MCO (formulation 1.1) represents a good lower bound for the MDVRP because the average deviation between the objective function values for both problems is $0.9 \%$, with a maximum of $3 \%$ (see Table 5 ). Likewise, the MDVRP-MCO is also a good lower bound for the MP-MDVRP given that the average deviation between both problems is $1.7 \%$. Although this is a higher percentage than for the MDVRP, it is still a small deviation.

An upper bound for these problems (MDVRP and MP-MDVRP) is the final solution provided by the decomposition method. The deviation between the lower and upper bounds obtained for each test instance is summarized in Table 5. Considering the MDVRP, the maximum deviation is $3.7 \%$ with an average of $1.2 \%$, representing a tight range. Regarding the MP-MDVRP, the gaps are slightly higher, with a maximum of $6.0 \%$ and an average of $2.2 \%$.

These results show that the decomposition method is effective in solving the problem in this study because it provides a good (or even optimal) solution in much less time than the monolithic models solved by the CPLEX solver. 
Table

Comparison between the optimal and the decomposition method values for service areas by recyclable material and by depot.

\begin{tabular}{|c|c|c|c|c|c|c|c|c|c|c|c|c|c|c|c|}
\hline \multirow[t]{3}{*}{ Problem } & \multirow[t]{3}{*}{$\left|V_{c}\right|$} & \multicolumn{7}{|c|}{ Service areas by recyclable material } & \multicolumn{7}{|c|}{ Service areas by depot } \\
\hline & & \multicolumn{3}{|c|}{ MDVRP@ Monolithic Model } & \multicolumn{2}{|c|}{ MDVRP @ Decomp. Method } & \multicolumn{2}{|c|}{ Deviation } & \multicolumn{3}{|c|}{ MP-MDVRP @ Monolithic Model } & \multicolumn{2}{|c|}{ MP-MDVRP @ Decomp. Method } & \multicolumn{2}{|c|}{ Deviation } \\
\hline & & OFV & Time (s) & GAP (\%) & OFV & Time (s) & OFV (\%) & Time (\%) & OFV & Time (s) & GAP (\%) & OFV & Time (s) & $\mathrm{OFV}(\%)$ & Time (\%) \\
\hline 1 & 6 & 886 & 1 & 0.0 & 887 & 1 & 0.1 & 0.0 & 892 & 6 & 0.0 & 899 & 2 & 0.8 & -90.0 \\
\hline 2 & 8 & 853 & 6 & 0.0 & 860 & 1 & 0.8 & -81.7 & 860 & 26 & 0.0 & 860 & 2 & 0.0 & -95.8 \\
\hline 3 & 10 & 650 & 8 & 0.0 & 650 & 2 & 0.0 & -80.0 & 650 & 660 & 0.0 & 650 & 3 & 0.0 & $\begin{array}{l}-99.8 \\
\end{array}$ \\
\hline 4 & 11 & 1178 & 17 & 0.0 & 1178 & 2 & 0.0 & -90.6 & 1186 & 16142 & 0.0 & 1186 & 3 & 0.0 & -100.0 \\
\hline 5 & 12 & 698 & 26 & 0.0 & 698 & 4 & 0.0 & -84.6 & 698 & 15684 & 0.0 & 698 & 5 & 0.0 & -100.0 \\
\hline 6 & 13 & 1193 & 29 & 0.0 & 1208 & 4 & 1.3 & -85.5 & 1193 & 28800 & 6.9 & 1193 & 5 & 0.0 & -100.0 \\
\hline 7 & 14 & 851 & 248 & 0.0 & 851 & 6 & 0.0 & -97.6 & 872 & 28800 & 5.0 & 889 & 7 & 2.0 & -100.0 \\
\hline 8 & 15 & 1007 & 33 & 0.0 & 1008 & 6 & 0.2 & -81.2 & 1046 & 28800 & 1.1 & 1046 & 7 & 0.0 & -100.0 \\
\hline 9 & 16 & 1489 & 785 & 0.0 & 1489 & 8 & 0.0 & -99.0 & 1509 & 28800 & 4.7 & 1509 & 9 & 0.0 & -100.0 \\
\hline 10 & 17 & 1523 & 802 & 0.0 & 1525 & 13 & 0.1 & -98.4 & 1541 & 28800 & 7.0 & 1574 & 14 & 2.1 & -100.0 \\
\hline 11 & 18 & 1228 & 4566 & 0.0 & 1228 & 17 & 0.0 & -99.6 & 1228 & 28800 & 6.3 & 1228 & 19 & 0.0 & -99.9 \\
\hline 12 & 19 & 1640 & 1764 & 0.0 & 1640 & 45 & 0.0 & -97.4 & 1641 & 28800 & 6.0 & 1641 & 47 & 0.0 & -99.8 \\
\hline 13 & 19 & 1826 & 8113 & 0.0 & 1850 & 33 & 1.3 & -99.6 & 1833 & 28800 & 5.8 & 1833 & 35 & 0.0 & -99.9 \\
\hline 14 & 20 & 2037 & 86400 & 6.0 & 2052 & 93 & 0.7 & -99.9 & 2062 & 28800 & 13.5 & 2081 & 96 & 0.9 & -99.7 \\
\hline 15 & 20 & 2179 & 15986 & 0.0 & 2190 & 14 & 0.5 & -99.9 & 2198 & 28800 & 6.8 & 2243 & 16 & 2.1 & -100.0 \\
\hline Average & - & - & 7919 & 0.4 & _ & 17 & 0.3 & -86.3 & - & 21368 & 4.2 & - & 18 & 0.5 & -99.0 \\
\hline Minimum & - & - & 1 & 0.0 & - & 1 & 0.0 & -99.9 & - & 6 & 0.0 & - & 2 & 0.0 & -100.0 \\
\hline Maximum & - & - & 86400 & 6.0 & - & 93 & 1.3 & 0.0 & - & 28800 & 13.5 & - & 96 & 2.1 & -90.0 \\
\hline
\end{tabular}

$\mathrm{OFV}=$ objective function value

GAP $=($ Upper Bound CPLEX-Lower Bound CPLEX $) /$ Upper Bound CPLEX $\times 100$

Deviation $=($ Decomp. Method Value/Monolithic Model Value -1$) \times 100$ 
Comparisons between MDVRP-MCO, MDVRP and MP-MDVRP.

\begin{tabular}{|c|c|c|c|c|}
\hline & \multicolumn{2}{|l|}{ Lower bound deviation } & \multicolumn{2}{|l|}{ Upper bound deviation } \\
\hline & $\begin{array}{l}\text { MDVRP-MCO @ Cplex vs. } \\
\text { MDVRP @ Cplex (\%) }\end{array}$ & $\begin{array}{l}\text { MDVRP-MCO @ Cplex vs. MP- } \\
\text { MDVRP @ Cplex (\%) }\end{array}$ & $\begin{array}{l}\text { MDVRP-MCO @ Cplex vs. MDVRP } \\
\text { @ Decomp.Met. (\%) }\end{array}$ & $\begin{array}{l}\text { MDVRP-MCO @ Cplex vs. MP- } \\
\text { MDVRP @ Decomp.Met. (\%) }\end{array}$ \\
\hline Average & 0.9 & 1.7 & 1.2 & 2.2 \\
\hline Minimum & 0.0 & 0.0 & 0.0 & 0.0 \\
\hline Maximum & 3.0 & 4.3 & 3.7 & 6.0 \\
\hline
\end{tabular}

\section{Case Study - A real recyclable waste collection system}

The recyclable waste collection system considered in this study is responsible for collecting three packaging materials in seven municipalities in mainland Portugal. The system operates five depots, with one of them also acting as a sorting station. The system has a total of 1622 bins clustered on 230 collections sites located in localities or isolated places. A collection site aggregates one or more bins of one or more recyclable materials. For example, collection site "1" is a locality with 4 glass bins, 3 paper bins and 3 plastic/metal bins. Therefore, the distance traveled and the time spent within a collection site considers the average distance between bins $(S=650 \mathrm{~m})$, the speed within localities $(v w=500 \mathrm{~m} / \mathrm{min})$ and the average time spent to collect each bin $(U=7 \mathrm{~min}$ ). The packaging materials have different collection frequencies. Glass is only collected once a month, whereas paper is collected four times a month and plastic/metal is collected twice a month. Because the lowest collection frequency is once a month, a planning horizon of four weeks (i.e., 20 working days) will be considered.

The company is presently evaluating the restructuring of the current service areas and the associated vehicle routes, with the goal of reducing the variable costs and the $\mathrm{CO}_{2}$ emissions of the existing collection system. Resizing the vehicle fleet is also under analysis so that the number of vehicles available for inbound transportation will not be constrained. The minimum number of required vehicles is an output of the model and not a constraint. Thus, parameter $\lambda$ in formulations 1.1 and 2 and the set $K$ in formulation 1.2 have a sufficiently large cardinality so as not to limit the solution.

The company's average distance traveled per month is approximately $30,000 \mathrm{~km}$ and has an estimated cost per kilometer (including fuel consumption, driver costs, vehicle depreciation, insurance and maintenance) of $0.70 €$. The variable cost associated with the current collection system is of $21,000 €$ per month. The $\mathrm{CO}_{2}$ emissions for the current collection system are estimated to be $1.1 \mathrm{~kg} / \mathrm{km}$, representing a total of $33,000 \mathrm{~kg}$ of $\mathrm{CO}_{2}$ per month.

The collection capacity of the vehicles is $4,500 \mathrm{~kg}$ for glass $\left(Q_{m 1}\right), 3400 \mathrm{~kg}$ for paper $\left(Q_{m 2}\right)$ and $600 \mathrm{~kg}$ for plastic/metal $\left(Q_{m 3}\right)$. The inbound transportation vehicles have a drag coefficient of $0.77\left(C d_{i n b}\right)$, a frontal surface area of $6.5 \mathrm{~m}^{2}\left(O_{i n b}\right)$, a curb (empty) weight of $8,000 \mathrm{~kg}\left(c w_{i n b}\right)$ and an engine displacement of 9 liters $\left(B_{i n b}\right)$. The vehicle capacity for the outbound transportation is $12,000 \mathrm{~kg}$ for glass $\left(Q T_{m 1}\right), 4,000 \mathrm{~kg}$ for paper $\left(Q T_{m 2}\right)$ and 2,000 kg for plastic/metal $\left(Q T_{m 3}\right)$. These vehicles have a drag coefficient of $0.86\left(C d_{\text {outb }}\right)$, a frontal surface area of $8 \mathrm{~m}^{2}\left(O_{\text {outb }}\right)$, a curb weight of $14,000 \mathrm{~kg}\left(\mathrm{cw}_{\text {outb }}\right)$ and an engine displacement of 11.7 liters $\left(B_{\text {outb }}\right)$. Due to the vehicle characteristics, we have assumed an engine efficiency of $0.45(\eta)$, a vehicle drivetrain efficiency of $0.4(\varepsilon)$, an engine friction factor of $0.2 \mathrm{~kJ} / \mathrm{rev} / \mathrm{liter}(R)$ and an engine speed of 33.3(3) revolutions per second $(N)$ for both types of vehicles (inbound and outbound transportation).

The maximum time allowed for a working day is $480 \mathrm{~min}(T)$. The average time to unload the vehicle at the depot at the end of each route is $15 \mathrm{~min}(L)$. The average speed between collection sites is $1000 \mathrm{~m} / \mathrm{min}(v b)$ and we assume an

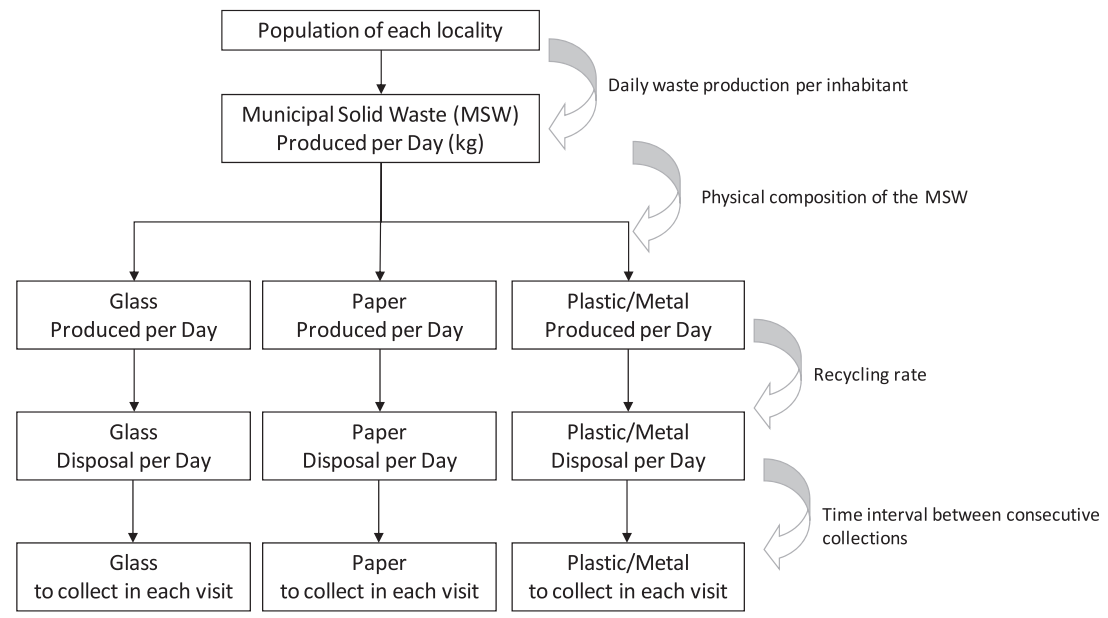

Fig. 5. Procedure for estimating the amount of material to collect at each collection site. 
acceleration of $0(u)$. The road slope $(\theta)$ is always $0^{\circ}$ because the geographical area in the study is flat. We assume a rolling coefficient ( $\mathrm{Cr}$ ) for concrete roads of 0.01 and an air density $(\rho)$ for an average of $20^{\circ} \mathrm{C}$ of $1.204 \mathrm{~kg} / \mathrm{m}^{3}$. The gravitational constant $(g)$ is $9.81 \mathrm{~m} / \mathrm{s}^{2}$.

The company has no record of the amounts collected by collection site or by route. To overcome this issue, we chose to use secondary data to estimate the collection amount at each site $\left(p_{i m}\right)$. The methodology used to compute $p_{i m}$ is illustrated in Fig. 5.

This procedure requires the population of each locality $i \in V_{c}$ and the daily waste production average ratio per inhabitant as input data. The daily municipal solid waste (MSW) produced is thus obtained. By applying the physical composition of the MSW, one can estimate the amount of glass, paper and plastic/metal produced per day. Not all of the produced amounts are disposed of at specific recyclable bins. Therefore, we applied a recycling rate for each material. Because there is no daily collection, a time interval is applied to each material in determining the amount to be collected at each visit $\left(p_{\text {im }}\right)$.

To accomplish the company's goals, six scenarios are studied considering different service area configurations and two different objectives (see Table 6): (A) maintain the current service areas, i.e., respecting the municipalities' boundaries but restructuring the current vehicle routes, considering an (A1) economic objective and an (A2) environmental objective; (B) restructure service areas by recyclable material, considering an (B1) economic objective and an (B2) environmental objective; and (C) restructure service areas by depot, considering an (C1) economic objective and an (C2) environmental objective. For each scenario, a different problem must be solved as summarized in Table 6 .

\subsection{Main results}

When solving each scenario through the decomposition solution method, different service areas and, consequently, different vehicle routes are obtained. Fig. 6 shows the service areas obtained for each scenario.

In Scenario A, the service areas are presently in use by the company and respect municipal boundaries. The number of collection sites belonging to each depot is relatively balanced. For Scenarios B and C, the service areas substantially change from the current ones. In Scenario B, three service areas are defined. Thus, each depot must manage three different intervention areas, depending on the material. In comparing the service areas by recyclable material, it can be observed that they are quite different from each other. Either at B1 or B2, plastic/metal generates more balanced service areas among the depots (all depots collect from a significant number of sites), whereas paper generates more unbalanced service areas (depots 232 and 234 provide little or no contribution; at B2, depot 231 also provides little contribution).

In comparing the service areas between Scenarios B1 and B2, some differences can be identified. In the latter, more collection sites are assigned to depot 235 (159 versus 149 for glass, 153 versus 122 for paper and 72 versus 50 for plastic/metal), which is also the sorting station. The energy required for outbound transportation is significantly higher than that for inbound transportation (approximately 50\% higher). Therefore, with the energy-minimization objective function (Scenario B2), the outbound transportation is minimized and more collection sites are assigned to the sorting station. At the farthest depot from the sorting station (depot 234), no sites are assigned for paper.

Scenario $C$ assumes that each depot has one common service area for all recyclable materials, as in Scenario A. In comparing the current service areas with the ones proposed by Scenario C, significant differences are observed. Two depots stand out (depots 233 and 235), decreasing or eliminating the outbound transportation, whereas the remaining three see their importance diminished. This observation indicates that to collect the amounts considered, the system could operate with a smaller number of depots, thus reducing the variable costs by 6\% (comparing Scenario C1 with A1), in addition to the savings from removing the fixed costs associated with closing some facilities. In comparing Scenarios C1 and C2, the sorting station collects 84\% of the total collection sites in Scenario C2 and 68\% in Scenario C1. Depot 231 is responsible for the collection of 18 sites in Scenario C1 but only two sites in Scenario C2.

As shown in Table 7, maintaining the current service areas (Scenarios A1 and A2) is the situation with the highest distance, cost and $\mathrm{CO}_{2}$ emissions. In comparing $\mathrm{A} 1$ with $\mathrm{A} 2$, the total distance increases by only $0.05 \%$ with the energy-minimization objective (Scenario $\mathrm{A} 2$ ), but the $\mathrm{CO}_{2}$ emissions decrease by $0.56 \%$. When restructuring the service areas by recyclable

Table 6

Scenarios in study.

\begin{tabular}{|c|c|c|c|}
\hline \multirow{2}{*}{$\begin{array}{l}\text { Objective } \\
\text { function }\end{array}$} & \multicolumn{3}{|l|}{ Service area configuration } \\
\hline & Maintain current service areas (A) & By recyclable material (B) & By depot (C) \\
\hline \multicolumn{4}{|c|}{ Economic (1) } \\
\hline & Scenario A1 & Scenario B1 & Scenario C1 \\
\hline & $\begin{array}{l}\text { VRP for each depot and each material with distance- } \\
\text { minimization objective }\end{array}$ & $\begin{array}{l}\text { MDVRP for each material with distance- } \\
\text { minimization objective }\end{array}$ & $\begin{array}{l}\text { MP-MDVRP with distance- } \\
\text { minimization objective }\end{array}$ \\
\hline \multicolumn{4}{|c|}{ Envirnomental (2) } \\
\hline & Scenario A2 & Scenario B2 & Scenario C2 \\
\hline & $\begin{array}{l}\text { VRP for each depot and each material with energy- } \\
\text { minimization objective }\end{array}$ & $\begin{array}{l}\text { MDVRP for each material with energy- } \\
\text { minimization objective }\end{array}$ & $\begin{array}{l}\text { MP-MDVRP with energy- } \\
\text { minimization objective }\end{array}$ \\
\hline
\end{tabular}




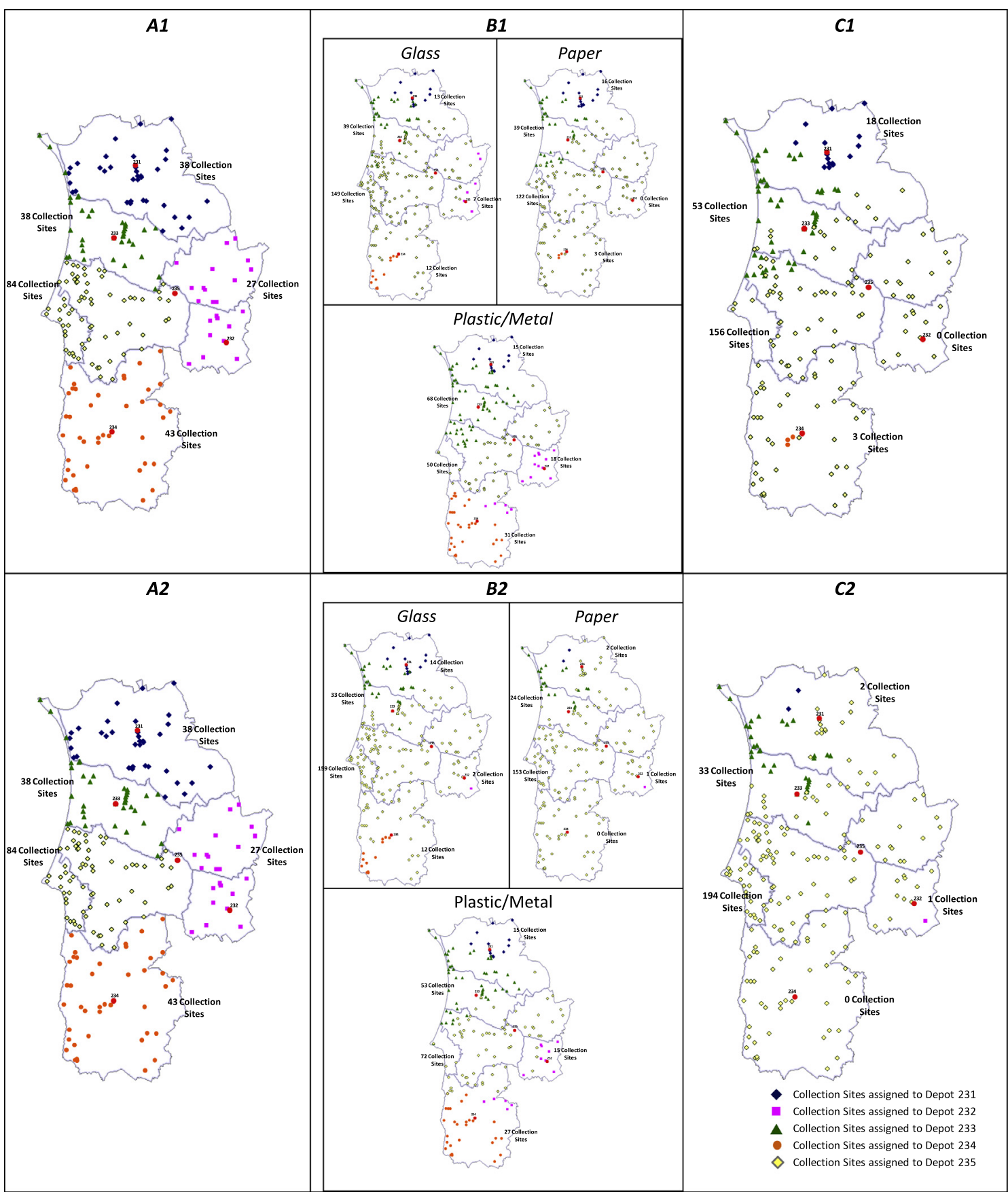

Fig. 6. Service areas by scenario.

material (Scenarios B1 and B2), the total distance decreases by $11 \%$ comparing Scenario B1 to Scenario A1; the outbound distance decreases by $47 \%$ because the service area of the sorting station is larger, and the inbound distance increases by $1 \%$. When restructuring by depot (Scenarios C1 and C2), the total distance increases by $5 \%$ comparing $\mathrm{C} 1$ to $\mathrm{B} 1$ because it is mandatory that all recyclable materials are collected from the same depot.

The working hours represent the number of hours needed to complete the inbound routes for each scenario over the assumed planning horizon (20 working days). Considering that each vehicle has a maximum usage of $160 \mathrm{~h}$ over the planning horizon ( 20 working days $\times 8 \mathrm{~h}$ ), we compute the minimum number of vehicles needed. All scenarios require a minimum of six vehicles. 
Table 7

Numerical results for each scenario.

\begin{tabular}{|c|c|c|c|c|c|c|}
\hline & \multicolumn{2}{|c|}{ Maintain current service areas } & \multicolumn{2}{|c|}{ Service areas by Recycl. Mat. } & \multicolumn{2}{|c|}{ Service areas by Depot } \\
\hline & $\mathrm{A} 1$ & A2 & B1 & B2 & $\mathrm{C} 1$ & $\mathrm{C} 2$ \\
\hline \multicolumn{7}{|l|}{ Distance ( $\mathrm{km})$} \\
\hline Inbound & 19,793 & 19,807 & 19,989 & 22,150 & 21,785 & 25,429 \\
\hline Outbound & 6279 & 6279 & 3305 & 1988 & 2620 & 318 \\
\hline Total & 26,072 & 26,086 & 23,294 & 24,138 & 24,405 & 25,747 \\
\hline Variable cost $(€)$ & 18250 & 18,260 & 16,306 & 16,897 & 17,084 & 18,023 \\
\hline \multicolumn{7}{|l|}{$\mathrm{CO}_{2}$ Emissions ( $\mathrm{kg}$ ) } \\
\hline Inbound & 19,125 & 18,963 & 19,316 & 21,239 & 21,049 & 24,566 \\
\hline Outbound & 9611 & 9611 & 5010 & 2783 & 4016 & 490 \\
\hline Total & 28,736 & 28,574 & 24,326 & 24,022 & 25,065 & 25,056 \\
\hline Working hours (h) & 848 & 856 & 865 & 886 & 884 & 948 \\
\hline Minimum vehicles needed & 6 & 6 & 6 & 6 & 6 & 6 \\
\hline
\end{tabular}
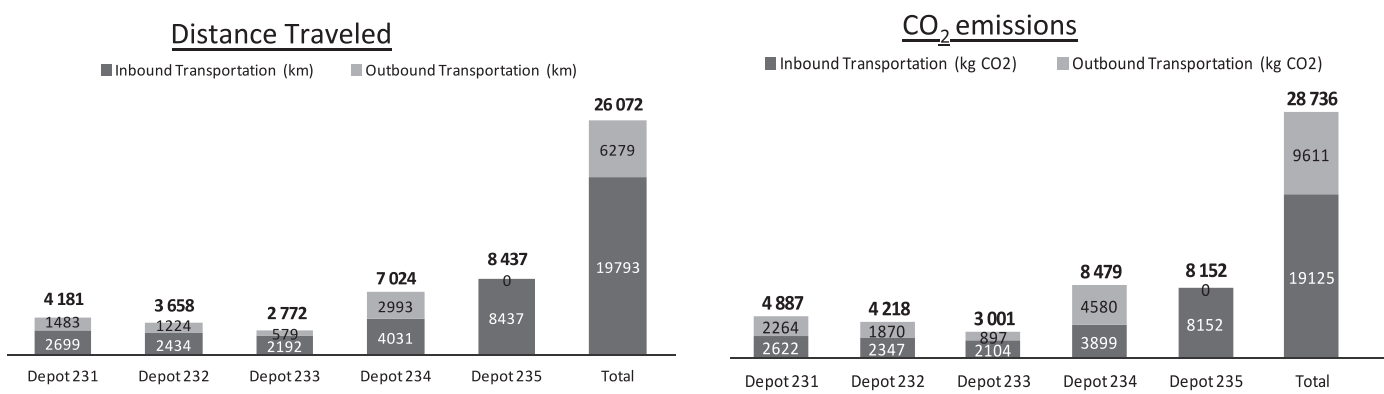

Fig. 7. Distance traveled and $\mathrm{CO}_{2}$ emissions by depot in Scenario $\mathrm{A} 1$.

A more detailed look at some solutions, the results obtained for depot 234 in Scenario A1 (see Fig. 7) show that it has the largest amount of $\mathrm{CO}_{2}$ emissions but not the longest distance traveled. Furthermore, this is the only depot where the outbound emissions exceed the inbound ones. It should be noted that this depot is the one located the farthest from the sorting station, and the outbound transportation has higher emissions than the inbound transportation (the ratio of $\mathrm{CO}_{2}$ per $\mathrm{km}$ is $0.97 \mathrm{~kg} / \mathrm{km}$ for inbound transportation and $1.53 \mathrm{~kg} / \mathrm{km}$ for outbound transportation).

When solving the problems with an energy-minimization objective, different service areas are created with more sites assigned to the sorting station to avoid outbound transportation. Moreover, route configuration also changes. This model seeks solutions in which vehicles travel a shorter distance with heavy loads. Therefore, the heaviest collection sites are collected later in the route or more routes with smaller loads are defined. Fig. 8 presents the routes defined with a

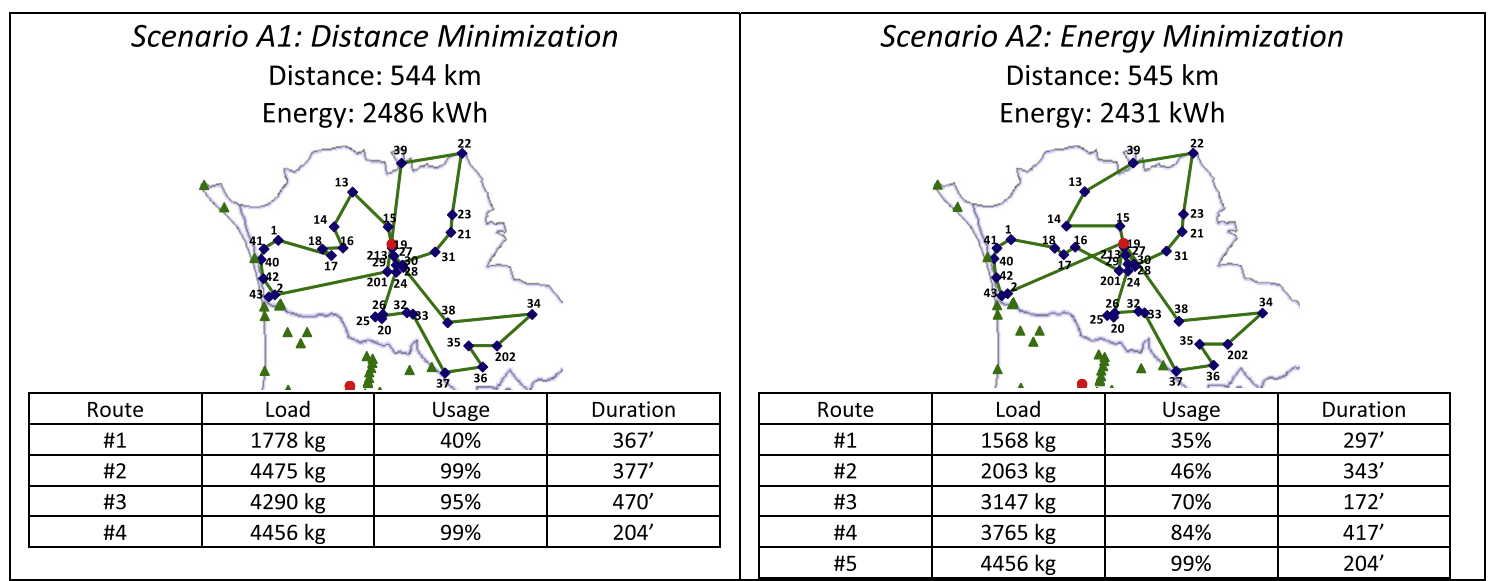

Fig. 8. Route solution with distance-minimizing versus energy-minimizing objective function for glass in depot 231 . 


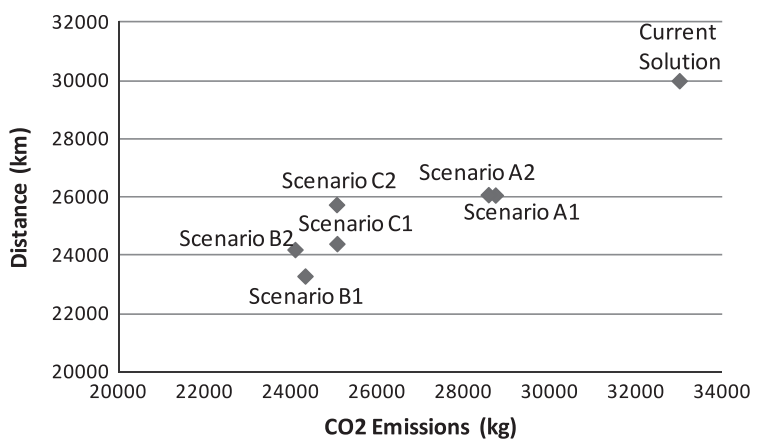

Fig. 9. Performance in the six scenarios under both objectives (distance and $\mathrm{CO}_{2}$ emissions).

Table 8

Computational results for Step 2 in Scenario A.

\begin{tabular}{|c|c|c|c|c|c|c|c|c|c|}
\hline & \multicolumn{3}{|l|}{ Glass } & \multicolumn{3}{|l|}{ Paper } & \multicolumn{3}{|l|}{ Plastic/metal } \\
\hline & OFV (km) & Time (s) & GAP (\%) & OFV (km) & Time (s) & GAP (\%) & OFV (km) & Time (s) & GAP (\%) \\
\hline \multicolumn{10}{|l|}{ Scenario A1 } \\
\hline Depot 231 & 544 & 3600 & 2.9 & 2409 & 198 & 0.0 & 1228 & 3164 & 0.0 \\
\hline Depot 232 & 462 & 77 & 0.0 & 1979 & 55 & 0.0 & 1217 & 36 & 0.0 \\
\hline Depot 233 & 465 & 3600 & 1.8 & 1521 & 16 & 0.0 & 785 & 23 & 0.0 \\
\hline Depot 234 & 889 & 3600 & 4.5 & 3843 & 3600 & 1.9 & 2292 & 3600 & 2.7 \\
\hline Depot 235 & 1305 & 3600 & 2.2 & 3847 & 3600 & 2.1 & 3285 & 3600 & 2.3 \\
\hline \multirow[t]{2}{*}{ Total } & 3665 & 14477 & - & 13599 & 7469 & - & 8808 & 10423 & - \\
\hline & OFV (kW h) & Time (s) & GAP (\%) & OFV (kW h) & Time (s) & GAP (\%) & OFV (kW h) & Time $(\mathrm{s})$ & GAP (\%) \\
\hline \multicolumn{10}{|l|}{ Scenario A2 } \\
\hline Depot 231 & 2431 & 3600 & 3.0 & 10951 & 3600 & 0.6 & 5073 & 179 & 0.0 \\
\hline Depot 232 & 2108 & 505 & 0.0 & 8956 & 191 & 0.0 & 4956 & 26 & 0.0 \\
\hline Depot 233 & 1994 & 3600 & 3.5 & 6298 & 195 & 0.0 & 3065 & 29 & 0.0 \\
\hline Depot 234 & 4339 & 3600 & 3.1 & 18120 & 3600 & 2.0 & 9743 & 3600 & 1.8 \\
\hline Depot 235 & 4958 & 3600 & 2.2 & 14432 & 3600 & 4.4 & 11652 & 3600 & 2.5 \\
\hline Total & 15,830 & 14,905 & - & 58,757 & 11,186 & - & 34,489 & 7434 & - \\
\hline
\end{tabular}

Table 9

Computational results for formulation 1.1 in Scenario B.

\begin{tabular}{|c|c|c|c|c|c|c|c|}
\hline \multicolumn{4}{|c|}{ Scenario B1: distance minimization } & \multicolumn{4}{|c|}{ Scenario B2: energy minimization } \\
\hline Recyclable material & $\mathrm{OFV}(\mathrm{km})$ & Time $(\mathrm{s})$ & GAP & Recyclable material & OFV (kW h) & Time $(s)$ & GAP \\
\hline Glass & 3381 & 28,800 & $7.9 \%$ & Glass & 14,330 & 28,800 & $9.2 \%$ \\
\hline Paper & 11,595 & 28,800 & $7.7 \%$ & Paper & 45,236 & 28,800 & $11.1 \%$ \\
\hline Plastic/metal & 8047 & 28,800 & $4.6 \%$ & Plastic/metal & 31,299 & 28,800 & $4.0 \%$ \\
\hline Total & 23,023 & 86,400 & - & Total & 90,865 & 86,400 & - \\
\hline
\end{tabular}

distance-minimization versus an energy-minimization objective function for glass at depot 231 for Scenario A. When the objective is to minimize distance, four routes are defined, with three of them above $95 \%$ of the capacity usage rate. This solution requires a total of $544 \mathrm{~km}$ and $2486 \mathrm{~kW}$ h of energy (corresponding to $651 \mathrm{~kg}$ of $\mathrm{CO}_{2}$ emissions). When the objective is energy minimization, five routes are defined, four of which are below $84 \%$ of the capacity usage rate. This solution implies one extra kilometer and 15 extra minutes to unload the fifth route, but reduces $\mathrm{CO}_{2}$ emissions by $14 \mathrm{~kg}$ (approximately $2.2 \%$ ).

Fig. 9 shows the performance of the six scenarios based on both objectives: distance and $\mathrm{CO}_{2}$ emissions.

The scenario with the shortest traveled distance is Scenario B1, and the lowest $\mathrm{CO}_{2}$ emissions occur in Scenario B2. If the company chooses to maintain their current service areas, distance and emissions savings of $13 \%$ can be achieved with routing optimization (Scenarios A1 and A2). However, if the company chooses to operate restructured service areas by depot, annual distance savings of $19 \%$ are obtained with Scenario C1 and emissions savings of $24 \%$ with Scenario C2. In addition, if the company chooses to change its operational structure and operates with service areas by recyclable material (Scenario B), the distance savings can reach $22 \%$ and the emissions savings can reach $27 \%$ compared to the current operating solution. 
Table 10

Computational results for Step 2 in Scenario B.

\begin{tabular}{|c|c|c|c|c|c|c|c|c|c|}
\hline & \multicolumn{3}{|l|}{ Glass } & \multicolumn{3}{|l|}{ Paper } & \multicolumn{3}{|l|}{ Plastic/metal } \\
\hline & OFV (km) & Time (s) & GAP (\%) & OFV (km) & Time (s) & GAP (\%) & OFV (km) & Time (s) & GAP (\%) \\
\hline \multicolumn{10}{|l|}{ Scenario B1 } \\
\hline Depot 231 & 247 & 2 & 0.0 & 1290 & 0.3 & 0.0 & 531 & 1 & 0.0 \\
\hline Depot 232 & 161 & 0.2 & 0.0 & - & - & - & 676 & 3 & 0.0 \\
\hline Depot 233 & 532 & 3600 & 5.9 & 1604 & 6 & 0.0 & 2749 & 3600 & 2.4 \\
\hline Depot 234 & 280 & 2 & 0.0 & 419 & 0.1 & 0.0 & 1725 & 414 & 0.0 \\
\hline Depot 235 & 2317 & 3600 & 2.1 & 8283 & 3600 & 7.6 & 2481 & 3600 & 2.0 \\
\hline \multirow[t]{2}{*}{ Total } & 3536 & 7204 & - & 11595 & 3606 & - & 8163 & 7618 & - \\
\hline & OFV (kW h) & Time (s) & GAP (\%) & OFV (kW h) & Time (s) & GAP (\%) & OFV (kW h) & Time (s) & GAP (\%) \\
\hline \multicolumn{10}{|l|}{ Scenario B2 } \\
\hline Depot 231 & 1174 & 0.8 & 0.0 & 606 & 0.2 & 0.0 & 2304 & 0.6 & 0.0 \\
\hline Depot 232 & 279 & 0.2 & 0.0 & 244 & 0.2 & 0.0 & 2281 & 1 & 0.0 \\
\hline Depot 233 & 1503 & 3600 & 6.7 & 3630 & 3.5 & 0.0 & 7919 & 3600 & 1.9 \\
\hline Depot 234 & 1477 & 3 & 0.0 & - & - & - & 6497 & 208 & 0.0 \\
\hline Depot 235 & 9996 & 3600 & 10.2 & 40931 & 3600 & 10.9 & 12,858 & 3600 & 3.9 \\
\hline Total & 14429 & 7204 & - & 45411 & 3604 & - & 31859 & 7410 & - \\
\hline
\end{tabular}

Table 11

Computational results for Step 2 in Scenario C.

\begin{tabular}{|c|c|c|c|c|c|c|c|c|c|}
\hline & \multicolumn{3}{|l|}{ Glass } & \multicolumn{3}{|l|}{ Paper } & \multicolumn{3}{|l|}{ Plastic/metal } \\
\hline & OFV (km) & Time (s) & GAP (\%) & OFV $(\mathrm{km})$ & Time (s) & GAP (\%) & OFV $(\mathrm{km})$ & Time (s) & GAP (\%) \\
\hline \multicolumn{10}{|l|}{ Scenario C1 } \\
\hline Depot 231 & 260 & 1 & 0.0 & 1290 & 0.3 & 0.0 & 592 & 1.5 & 0.0 \\
\hline Depot 232 & - & - & - & - & - & - & - & - & - \\
\hline Depot 233 & 679 & 3600 & 4.8 & 2246 & 4 & 0.0 & 1456 & 3600 & 2.2 \\
\hline Depot 234 & 96 & 0.1 & 0.0 & 419 & 0.1 & 0.0 & 219 & 0.1 & 0.0 \\
\hline Depot 235 & 2625 & 3600 & 2.3 & 7789 & 3600 & 7.6 & 6734 & 3600 & 6.2 \\
\hline \multirow[t]{2}{*}{ Total } & 3659 & 7201 & - & 11,744 & 3604 & - & 9002 & 7202 & - \\
\hline & OFV (kW h) & Time (s) & GAP (\%) & OFV (kW h) & Time (s) & GAP (\%) & OFV (kW h) & Time (s) & GAP (\%) \\
\hline \multicolumn{10}{|l|}{ Scenario C2 } \\
\hline Depot 231 & 146 & 0.3 & 0.0 & 606 & 0.5 & 0.0 & 294 & 0.7 & 0.0 \\
\hline Depot 232 & 59 & 0.3 & 0.0 & 244 & 0.6 & 0.0 & 118 & 0.2 & 0.0 \\
\hline Depot 233 & 1166 & 3600 & 9.2 & 3630 & 3 & 0.0 & 1970 & 178 & 0.0 \\
\hline Depot 234 & - & - & - & - & - & - & - & - & - \\
\hline Depot 235 & 13091 & 3600 & 9.4 & 40931 & 3600 & 10.9 & 33392 & 3600 & 4.9 \\
\hline Total & 14,461 & 7201 & - & 45,411 & 3604 & - & 35,775 & 3779 & - \\
\hline
\end{tabular}

\subsection{Computational results}

The decomposition method was implemented in GAMS 23.6 and solved through the CPLEX Optimizer 12.1.0 on an Intel Xeon CPU X5680 @ $3.33 \mathrm{GHz}$.

Because the service areas are already defined in Scenario A, the decomposition approach is reduced to Step 2. The computational results for Scenario A1 and A2 are shown in Table 8. The solution found in 13 out of 30 instances is the optimal one ( $0 \%$ gap). For the remaining instances, the model is not capable of proving the optimality within $1 \mathrm{~h}$, but lower gaps are achieved.

For Scenario B, Steps 1 and 2 are executed, but heuristic procedure 1.3 from Step 1 is not run. The computational results for formulation 1.1 for both scenarios B1 and B2 are presented in Table 9, with the computational time limited to $8 \mathrm{~h}$ $(28,800 \mathrm{~s})$. Solution optimality is not proven within the 8 -h limit, but the gap between the integer solution and the lower bound provided by CPLEX does not exceed $8 \%$ in Scenario B1. Higher gaps are obtained in Scenario B2, which may be explained by its higher complexity.

For formulation 1.2, all instances are solved to optimality in few minutes. In all, 48 instances are run, corresponding to the total of open routes generated in both scenarios for the three materials. The individual computational results are not shown.

The computational results from Step 2 for scenario B are shown in Table 10. This formulation is capable of proving optimality in 18 out of 28 instances. In two instances, the gap exceeds 10\%. These are the largest instances with 153 (depot 235 for paper) and 159 collection sites (depot 235 for glass).

For Scenario C, the decomposition method is fully applied. The results from formulations 1.1 and 1.2 are the same as those in Scenario B. Procedure 1.3 is executed in a few seconds. The 91 and 75 unclear sites for Scenario C1 and C2, respectively, are 
assigned to a depot according to the material with the highest collection frequency (i.e., paper). The computational results for Step 2 are shown in Table 11. This module is capable of proving optimality in 15 out of 24 instances.

\section{Conclusions}

This work addresses the solution of a multi-product, multi-depot vehicle routing problem, which often characterizes real logistics networks such as recyclable waste collection systems.

The first contribution of this paper to the logistics literature is the development of a mathematical model approach that integrates decisions for service areas and vehicle route definitions in logistics systems with multiple depots and multiple products while considering inbound and outbound transportation flows. This comprehensive model allows for holistic planning, ensuring better overall solutions in terms of costs.

The second contribution of the paper is the extension of the developed formulation to account for a new objective function when planning logistics systems-the minimization of $\mathrm{CO}_{2}$ emissions. The proposed model addresses the increasing concerns of transportation companies and allows for a comparison with the distance-minimization objective-a proxy for the economic goal. Different logistic solutions are obtained when running both models. $\mathrm{The}^{\mathrm{CO}} \mathrm{C}_{2}$ emissions-minimizing model produces service areas with more sites assigned to the sorting station to minimize the outbound transportation. Furthermore, route topology also changes as the model seeks solutions in which the vehicles travel a shorter distance with heavy loads, with the heaviest collection sites collected later in the route. More routes with lighter loads can be obtained, but the final results regarding total distance traveled and $\mathrm{CO}_{2}$ emissions for the real case study did not differ substantially. These results allow us to conclude that the distance traveled has a major effect on $\mathrm{CO}_{2}$ emissions. Therefore, when the distance is minimized (a proxy for the economic goal), it simultaneously contributes to mitigate the negative environmental impact of transportation.

The third contribution of this paper is the development of a flexible decomposition solution method capable of solving three different problems and allowing for the study of alternative scenarios regarding service area configurations. First, the proposed method solves a MP-MDVRP, leading to a scenario in which the service areas are defined by depot (Scenario C). Second, it solves the MDVRP for each material, leading to a scenario in which the service areas are defined by recyclable material (Scenario B). Third, it solves the VRP when the service areas are previously defined (Scenario A). The results of the effectiveness tests show that the decomposition solution method is able to produce good solutions (or even optimal ones) in less time than the mathematical formulations for the MP-MDVRP and MDVRP solved by the CPLEX solver.

Finally, this paper also contributes to practice because the decomposition solution method is applied to a real recyclable waste collection system where the company wants to restructure its current service areas and vehicle routes to decrease collection variable costs and $\mathrm{CO}_{2}$ emissions. When compared to the current solution, the obtained results allow for a significant reduction in both economic and environmental terms. A decrease in total distance traveled of $13 \%$ to $22 \%$ (depending on the scenario) is achieved, as well as a decrease in $\mathrm{CO}_{2}$ emissions of $13 \%$ to $27 \%$. The results also show that service areas defined by recyclable material (Scenario B) provide a solution with the shortest total distance and lowest $\mathrm{CO}_{2}$ emissions. The case in which service areas are defined by depot (Scenario $C$ ) is the second lowest, followed by Scenario A, in which service areas are pre-defined by municipal boundaries. However, it is important to mention that Scenario B, despite providing the better solution in terms of distance and $\mathrm{CO}_{2}$ emissions, implies a significant change in the company's operational structure because three different service areas for each depot must be managed.

Although this paper addresses important problems faced by real waste collection systems, other aspects still remain to be explored. As future work, it is important to consider the balancing of the working hours among depots because some scenarios showed imbalanced solutions. Furthermore, the developed model assumes a deterministic approach, in which the quantities to collect are known. However, there is some level of uncertainty regarding this parameter. This issue should also be a topic of future research, being stochastic models developed to cope with more realistic scenarios. Scheduling vehicle routes according to the collection frequencies should also be considered to explore a scenario with shared resources versus fixed resources. With respect to shared resources, open routes between the depots can be scheduled to pre-position the vehicles at different depots. Finally, another possible extension of the present work is to consider other green logistics practices (such as eco-driving, hybrid vehicles or greener fuels) in recyclable waste collection systems, thus making recycling even greener.

\section{Acknowledgments}

The authors sincerely appreciate the constructive comments and suggestions of the anonymous referee and gratefully acknowledge the valuable suggestions of Professor Wayne K. Talley. This work was partially supported by CEG-IST/IST/ UTL and CMA/FCT/UNL under the Project PEst-OE/MAT/UI0297/2011.

\section{References}

Amponsah, S.K., Salhi, S., 2004. The investigation of a class of capacitated arc routing problems: the collection of garbage in developing countries. Waste Management 24, 711-721. 
Angelelli, E., Speranza, M.G., 2002. The application of a vehicle routing model to a waste-collection problem: two case studies. Journal of the Operational Research Society 53 (9), 944-952.

Baldacci, R., Hadjiconstantinou, E., Mingozzi, A., 2004. An exact algorithm for the capacitated vehicle routing problem based on a two-commodity network flow formulation. Operations Research 52 (5), 723-738.

Baldacci, R., Mingozzi, A., 2009. A unified exact method for solving different classes of vehicle routing problems. Mathematical Programming 120 (2), 347380.

Barth, M., Scora, G., Younglove, T., 2004. Modal emissions model for heavy-duty diesel vehicles. Transportation Research Record: Journal of the Transportation Research Board 1880, 10-20.

Bautista, J., Fernandez, E., Pereira, J., 2008. Solving an urban waste collection problem using ants heuristics. Computers and Operations Research 35, 30203033.

Bektas, T., Laporte, G., 2011. The pollution-routing problem. Transportation Research Part B - Methodological 45 (8), $1232-1250$.

Beltrami, E.J., Bodin, L.D., 1974. Networks and vehicle routing for municipal waste collection. Networks 4, 65-94.

Benjamin, A.M., Beasley, J.E., 2010. Metaheuristics for the waste collection vehicle routing problem with time windows, driver rest period and multiple disposal facilities. Computers and Operations Research 37, 2270-2280.

Carbon Trust, 2010. Resources-conversion factors. <http://www.carbontrust.co.uk/cut-carbon-reduce-costs/calculate/carbon-footprinting/pages/ conversion-factors.aspx> (accessed 15.09.11).

Chao, I., Golden, B.L., Wasil, E., 1993. A new heuristic for the multi-depot vehicle routing problem that improves upon best-known solutions. American Journal of Mathematical and Management Sciences 13, 371-406.

Cordeau, J.F., Gendreau, M., Laporte, G., 1997. A tabu search heuristic for periodic and multi-depot vehicle routing problems. Networks 30 (2), $105-119$.

Craighill, A.L., Powell, J.C., 1996. Lifecycle assessment and economic evaluation of recycling: a case study. Resources Conservation and Recycling 17 (2), $75-$ 96.

Crevier, B., Cordeau, J.F., Laporte, G., 2007. The multi-depot vehicle routing problem with inter-depot routes. European Journal of Operational Research 176 (2), 756-773.

De Figueiredo, J.N., Mayerle, S.F., 2008. Designing minimum-cost recycling collection networks with required throughput. Transportation Research Part E Logistics and Transportation Review 44, 731-752.

Demir, E., Bektas, T., Laporte, G., 2011. A comparative analysis of several vehicle emission models for road freight transportation. Transportation Research Part D - Transport and Environment 16 (5), 347-357.

Dondo, R.G., Cerda, J., 2009. A hybrid local improvement algorithm for large-scale multi-depot vehicle routing problems with time windows. Computers and Chemical Engineering 33 (2), 513-530.

Erdogan, S., Miller-Hooks, E., 2012. A green vehicle routing problem. Transportation Research Part E - Logistics and Transportation Review 48 (1), $100-114$.

Golden, B.L., Magnanti, T.L., Nguyen, H.Q., 1977. Implementing vehicle routing algorithms. Networks 7 (2), $113-148$.

Ho, W., Ho, G.T.S., Ji, P., Lau, H.C.W., 2008. A hybrid genetic algorithm for the multi-depot vehicle routing problem. Engineering Applications of Artificial Intelligence 21 (4), 548-557.

Kara, I., Kara, B.Y., Yetis, M.K., 2007. Energy minimizing vehicle routing problem. In: Dress, A., Xu, Y., Zhu, B. (Eds.), Combinatorial Optimization and Applications, Lecture Notes in Computer Science. Springer, Berlin/Heidelberg, pp. 62-71, vol. 4616.

Kim, B.I., Kim, S., Sahoo, S., 2006. Waste collection vehicle routing problem with time windows. Computers and Operations Research 33, $3624-3642$.

Laporte, G., Nobert, Y., Arpin, D., 1984. Optimal solutions to capacitated multi-depot vehicle routing problems. Congressus Numerantium 44, 283-292.

Laporte, G., Nobert, Y., Taillefer, S., 1988. Solving a family of multi-depot vehicle-routing and location-routing problems. Transportation Science 22 (3), $161-172$.

Lee, D.-H., Dong, M., 2008. A heuristic approach to logistics network design for end-of-lease computer products recovery. Transportation Research Part E - Logistics and Transportation Review 44, 455-474.

Lim, A., Wang, F., 2005. Multi-depot vehicle routing problem: a one-stage approach. Ieee Transactions on Automation Science and Engineering 2 (4), 397-402.

McLeod, F.N., Cherrett, T.J., Waterson, B.J., 2011. The scope for joint household/commercial waste collections: a case study. International Journal of Logistics-Research and Applications 14, 399-411.

Paksoy, T., Bektas, T., Ozceylan, E., 2011. Operational and environmental performance measures in a multi-product closed-loop supply chain. Transportation Research Part E - Logistics and Transportation Review 47, 532-546.

Palmer, A., 2007. The development of an integrated routing and carbon dioxide emissions model for goods vehicles. PhD thesis, Cranfield University, School of Management.

Parthanadee, P., Logendran, R., 2006. Periodic product distribution from multi-depots under limited supplies. Iie Transactions 38 (11), $1009-1026$.

Pisinger, D., Ropke, S., 2007. A general heuristic for vehicle routing problems. Computers and Operations Research 34 (8), $2403-2435$.

Ramos, T., Gomes, M.I., Barbosa-Póvoa, A.P., 2013. Planning waste cooking oil collection systems. Waste Management 33 (8), $1691-1703$.

Renaud, J., Laporte, G., Boctor, F.F., 1996. A tabu search heuristic for the multi-depot vehicle routing problem. Computers and Operations Research 23 (3), 229-235.

Rubio, S., Chamorro, A., Miranda, F.J., 2008. Characteristics of the research on reverse logistics (1995-2005). International Journal of Production Research 46, $1099-1120$.

Salhi, S., Sari, M., 1997. A multi-level composite heuristic for the multi-depot vehicle fleet mix problem. European Journal of Operational Research 103 (1), 95-112.

Sbihi, A., Eglese, R.W., 2007. Combinatorial optimization and green logistics. 4or-a Quarterly Journal of Operations Research 5 (2), 99-116.

Schweiger, K., Sahamie, R., 2013. A hybrid tabu search approach for the design of a paper recycling network. Transportation Research Part E 50, 98-119.

Teixeira, J., Antunes, A.P., de Sousa, J.P., 2004. Recyclable waste collection planning - a case study. European Journal of Operational Research 158 (3), 543 554.

Tillman, F.A., Cain, T.M., 1972. Upperbound algorithem for single and multiple terminal delivery problem. Management Science Series A - Theory 18 (11), $664-682$.

Tung, D.V., Pinnoi, A., 2000. Vehicle routing-scheduling for waste collection in Hanoi. European Journal of Operational Research 125 (3), $449-468$.

Ubeda, S., Arcelus, F.J., Faulin, J., 2011. Green logistics at Eroski: a case study. International Journal of Production Economics 131 (1), $44-51$.

Zhang, R.Y., Yun, W.Y., Moon, I., 2009. A reactive tabu search algorithm for the multi-depot container truck transportation problem. Transportation Research Part E - Logistics and Transportation Review 45 (6), 904-914. 